\title{
Jet Bundles on Projective Space II
}

\section{Maakestad $\mathrm{H}^{*}$}

Tempelveien 112, 3475 Sætre i Hurum, Bærum, Norway

\begin{abstract}
In previous papers the structure of the jet bundle as $P$-module has been studied using different techniques. In this paper we use techniques from algebraic groups, sheaf theory, generliazed Verma modules, canonical filtrations of irreducible $\mathrm{SL}(V)$-modules and annihilator ideals of highest weight vectors to study the canonical filtration $U_{l}(\mathfrak{g}) L^{d}$ of the irreducible $\mathrm{SL}(V)$-module $\mathrm{H}^{0}\left(X, \mathcal{O}_{x}(d)\right)^{*}$ where $X=\mathbb{G}(m, m+n)$. We study $\mathrm{U},(\mathfrak{g}) L^{d}$ using results from previous papers on the subject and recover a well known classification of the structure of the jet bundle $\mathcal{P}^{\prime}(\mathcal{O}(d))$ on projective space $\mathbb{P}\left(V^{*}\right)$ as $P$-module. As a consequence we prove formulas on the splitting type of the jet bundle on projective space as abstract locally free sheaf. We also classify the $P$-module of the first order jet bundle $\mathcal{P}_{X}^{1}\left(\mathcal{O}_{X}(d)\right)$ for any $d$ $\geq 1$. We study the incidence complex for the line bundle $\mathcal{O}(d)$ on the projective line and show it is a resolution of the ideal sheaf of $I^{\prime}(\mathcal{O}(d))$ - the incidence scheme of $\mathcal{O}(d)$. The aim of the study is to apply it to the study of syzygies of discriminants of linear systems on projective space and grassmannians.
\end{abstract}

Keywords: Algebraic group; Jet bundle; Grassmannian; P-module; Generalized verma module; Higher direct image; Annihilator ideal; Canonical filtration; Discriminant; Koszul complex; Regular sequence; Resolution

\section{Introduction}

In a series of papers of Maakestad [1-4], the structure of the jet bundle as $P$-module has been studied using different techniques. In this paper we continue this study using techniques from algebraic groups, sheaf theory, generalized Verma modules, canonical filtrations of irreducible $\mathrm{SL}(V)$-modules and annihilator ideals of highest weight vectors and study the canonical filtration $\mathrm{U}_{1}(\mathfrak{g}) L^{d}$ of the $\mathrm{SL}(V)$-module $\mathrm{H}^{0}\left(X, \mathcal{O}_{X}(d)\right)^{*}$ where $X=\mathbb{G}(m, m+n)$ is the grassmannian of $m$-planes in an $m+n$-dimensional vector space. Using results obtained in studies of Maakestad [1] we classify $\mathrm{U}_{l}(\mathrm{~g}) L^{d}$ and as a corollary we recover a well known result on the structure of the jet bundle $\mathcal{P}^{\prime}(\mathcal{O}(d))$ on $\mathcal{P}\left(V^{\star}\right)$ as $P$-module. As a consequence we get well known formulas on the splitting type of the jet bundle on projective space as abstract locally free sheaf. We also classify the $P$-module of the first order jet bundle $\mathcal{P}_{X}^{1}\left(\mathcal{O}_{X}(d)\right)$ on any grassmannian $X=\mathbb{G}(m, m+n)$ (Corollary 3.10).

In the first section of the paper we study the jet bundle $\mathcal{P}_{G / H}^{l}(\mathcal{E})$ of any locally free $G$-linearized sheaf $\varepsilon$ on any quotient $G / H$. Here $G$ is an affine algebraic group of finite type over an algebraically closed field $K$ of characteristic zero and $H \subseteq G$ is a closed subgroup. There is an equivalence of categories between the category of finite dimensional $H$-modules and the category of finite rank locally free $\mathcal{O}_{G / H}$-modules with a $G$-linearization. The main result of this section is Theorem 2.3 where we give a classification of the $H_{l}$-modules structure of the fiber $\mathcal{P}_{G / H}^{l}(\mathcal{E})(x)^{*}$ where $H_{l} \subseteq H$ is a Levi subgroup. Here $x G / H$ is the distinguished $K$-rational point defined by the identity $e \in G$. We also study the structure of $\mathcal{P}_{X}^{l}\left(\mathcal{O}_{X}(d)\right)(x)^{*}$ as $H_{l}$-module where $X=\mathbb{G}(m, m$ $+n)$ is the grassmannian of $m$-planes in an $m+n$-dimensional vector space (Corollary 2.5 and 2.8).

In the second section we study the canonical filtration $\mathrm{U}_{l}(\mathrm{~g}) L^{d}$ for the irreducible $\mathrm{SL}(V)$-module $\mathrm{H}^{0}(\mathbb{G}, \mathcal{O} \mathbb{G}(d))^{*}$. Here $\mathbb{G}=\mathbb{G}(m, m+n)$. We prove in Theorem 3.5 there is an isomorphism

$$
\mathrm{U}_{l}(\mathfrak{g}) L^{d} \cong L^{d-l} \otimes \operatorname{Sym}^{l}\left(\mathfrak{g} / \mathfrak{p}_{L} \otimes L\right)
$$

of $P$-modules when $\mathbb{G}=\mathbb{G}(1, n+1)=\mathcal{P}^{n}$ is projective $n$-space. As a result we recover in Corollary 3.6 the structure of the fiber $\mathcal{P}_{\mathbb{G}}^{l}\left(\mathcal{O}_{\mathbb{G}}(d)\right)(x)^{*}$ as $P$-module. This result was proved in another paper [5] using different techniques. We also recover in Corollary 3.8 a known formula on the structure of the jet bundle on projective space as abstract locally free sheaf $[2,6-10]$.

In the third section we study the incidence complex

$$
\wedge^{\bullet} \mathcal{O}_{\mathbb{P}\left(W^{*}\right)}(-1)_{Y} \otimes \mathcal{P}^{l}(\mathcal{O}(d))_{Y}^{*}
$$

of the line bundle $\mathcal{O}(d)$ on the projective line. Using Koszul complexes and general properties of jet bundles we prove it is a locally free resolution of the ideal sheaf of $I^{l}(\mathcal{O}(d))$ - the incidence scheme of $\mathcal{O}(d)$.

In Appendix A and B we study SL(V)-modules, automorphisms of $\mathrm{SL}(V)$-modules and give an elementary proof of the Cauchy formula.

Hence the paper initiates a general study of the canonical filtration $\mathrm{U}_{l}(\mathrm{~g}) L^{d}$ for any line bundle $\mathcal{O}(d)$ with $d \geq 1$ on any grassmannian $\mathbb{G}(m$, $m+n)$ as $P$-module. In Section 3 we show some of the complications arising in this study by giving explicit examples.

The study of the jet bundle $\mathcal{P}_{X}^{l}\left(\mathcal{O}_{X}(d)\right)$ of a line bundle $\mathcal{O}_{X}(d)$ on the grassmannian $X=\mathbb{G}(m, m+n)$ is motivated partly by its relationship with the discriminant $D^{l} \mathcal{O}_{X}(d)$ of the line bundle $\mathcal{O}_{X}(d)$. There is by studies of Maakestad [11] for all $1 \leq l<d$ an exact sequence of locally free $\mathcal{O}_{X}$-modules

$$
0 \rightarrow \mathcal{Q} \rightarrow \mathrm{H}^{0}\left(X, \mathcal{O}_{X}(d)\right) \otimes \mathcal{O}_{X} \rightarrow \mathcal{P}_{X}^{l}\left(\mathcal{O}_{x}(d)\right) \rightarrow 0
$$

giving rise to a diagram of maps of schemes

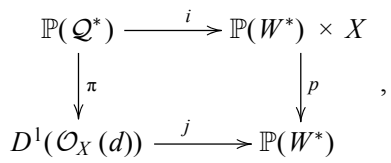

*Corresponding author: Maakestad H, Tempelveien 112, 3475 Sætre i Hurum Bærum, Norway, Tel: +4798457343; E-mail: h_maakestad@hotmail.com

Received October 21, 2015; Accepted November 24, 2015; Published December 01, 2015

Citation: Maakestad H (2015) Jet Bundles on Projective Space II. J Generalized Lie Theory Appl S2: 001. doi:10.4172/1736-4337.S2-001

Copyright: $\odot 2015$ Maakestad H. This is an open-access article distributed under the terms of the Creative Commons Attribution License, which permits unrestricted use, distribution, and reproduction in any medium, provided the original author and source are credited. 
Where $W=\mathrm{H}^{0}\left(X, \mathcal{O}_{X}(d)\right), \pi$ is the restriction of the projection map and $i, j$ are closed immersions. By definition $D^{l}\left(\mathcal{O}_{X}(d)\right):=\pi\left(\mathbb{P}\left(\mathcal{Q}^{*}\right)\right)$ is the schematic image of $\mathcal{P}\left(\mathcal{Q}^{*}\right)$ via $\pi$. The $K$-rational points of $\mathcal{P}\left(\mathcal{Q}^{*}\right)$ are pairs of $K$-rational points $(s, x)$ with the property that $T^{l}(x)(s)=0$ in $\mathcal{P}_{X}^{l}\left(\mathcal{O}_{X}(d)\right)(x)$. The scheme $\mathcal{P}\left(\mathcal{Q}^{*}\right)$ is the incidence scheme of the $l^{\prime}$ th Taylor morphism

$$
T^{l}: \mathrm{H}^{0}\left(X, \mathcal{O}_{X}(d)\right) \otimes \mathcal{O}_{X} \rightarrow \mathcal{P}_{X}^{l}\left(\mathcal{O}_{x}(d)\right) .
$$

The map $\pi$ is a surjective generically finite morphism between irreducible schemes. There is by literature of Maakestad [11] a Koszul complex of locally free sheaves on $Y=\mathcal{P}\left(W^{*}\right) \times X$

$$
\begin{aligned}
& 0 \rightarrow \mathcal{O}(-r)_{Y} \otimes \wedge^{r} \mathcal{P}_{X}^{l}\left(\mathcal{O}_{X}(d)\right)_{Y}^{*} \rightarrow \cdots \rightarrow \mathcal{O}(-1)_{Y} \otimes \mathcal{P}_{X}^{l}\left(\mathcal{O}_{X}(d)\right)_{Y}^{*} \rightarrow \\
& \mathcal{O}_{Y} \rightarrow \mathcal{O}_{\mathbb{P}\left(\mathcal{Q}^{*}\right)} \rightarrow 0
\end{aligned}
$$

which is a resolution of the ideal sheaf of $\mathcal{P}\left(\mathcal{Q}^{*}\right)$ when it is locally generated by a regular sequence. The complex 1 might give information on a resolution of the ideal sheaf of $D^{l}\left(\mathcal{O}_{X}(d)\right)$. A resolution of the ideal sheaf of $D^{l}\left(\mathcal{O}_{X}(d)\right)$ will give information on its syzygies. By literature of Maakestad [11] the first discriminant $D^{1}(\mathcal{O P}(d))$ on the projective line $\mathcal{P}=\mathcal{P}^{1}$ is the classical discriminant of degree $d$ polynomials, hence it is a determinantal scheme. By the results of Lascoux [12], we get an approach to the study of the syzygies of $D^{1}(\mathcal{O P}(d))$. Hence we get two approaches to the study of syzygies of discriminants of line bundles on projective space and grassmannians: One using Taylor maps, incidence schemes, jet bundles and generalized Verma modules. Another one using determinantal schemes.

\section{Jet Bundles on Quotients}

In this section we study the jet bundle of any finite rank $G$-linearized locally free sheaf $\mathcal{E}$ on the grassmannian $G / P=\mathbb{G}(m, m+n)$ as $P_{l}$ -module, where $P_{l} \subseteq P$ is a maximal linearly reductive subgroup.

Let $K$ be an algebraically closed field of characteristic zero and let $V$ be a $K$-vector space of dimension $n$. Let $H \subseteq G \subseteq \mathrm{GL}(V)$ be closed subgroups. The following holds: There is a quotient morphism

$$
\pi: G \rightarrow G / H
$$

and $G / H$ is a smooth quasi projective scheme of finite type over K. Moreover

$H \subseteq G$ is parabolic if and only if $G / H$ is projective.

For a proof refer to literature of Jantzen [13]. Let $X=G / H$ and let $\bmod ^{G}\left(\mathcal{O}_{G / H}\right)$ be the category of locally free $\mathcal{O}_{G / H}$-modules with a $G$-linearization. Let $\underline{\bmod }(H)$ be the category of finite dimensional $H$-modules. It follows from Jantzen [13], there is an exact equivalence of categories

$$
\underline{\bmod }(H) \cong \underline{\bmod }^{G}\left(\mathcal{O}_{G / H}\right) \text {. }
$$

Let $\mathcal{E} \in \underline{\bmod }^{G}\left(\mathcal{O}_{G / H}\right)$ be a locally free $\mathcal{O}_{G / H}$-module.

Let $Y=G / H \times G / H$ and $p, q: Y \rightarrow G / H$ be the canonical projection maps. The scheme $G / H$ is smooth and separated over $\operatorname{Spec}(K)$ hence the diagonal morphism

\section{$\Delta: G / H \rightarrow Y$}

is a closed immersion of schemes. Let $\mathcal{I} \subseteq \mathcal{O}_{Y}$ be the ideal of the diagonal and let $\mathcal{O}_{\Lambda^{l}}=\mathcal{O}_{Y} / \mathcal{I}^{l+1}$ be the structure sheaf of the $n^{\prime} t h$ infinitesimal neigborhood of the diagonal.

Definition 2.1. Let $\mathcal{E}$ be a locally free finite rank $\mathcal{O}_{G / H}$-module. Let

$$
\mathcal{P}_{G / H}^{l}(\mathcal{E})=p_{*}\left(\mathcal{O}_{\Delta^{l}} \otimes q^{*} \mathcal{E}\right)
$$

be the $l$ th jet bundle of $\mathcal{E}$.

Proposition 2.2. There is for all $l \geq 1$ an exact sequence of locally free $\mathcal{O}_{G / H}$-modules

$$
\begin{aligned}
& 0 \rightarrow \operatorname{Sym}^{l}\left(\Omega_{G / H}^{1}\right) \otimes \mathcal{E} \rightarrow \mathcal{P}_{G / H}^{l}(\mathcal{E}) \rightarrow^{\phi} \mathcal{P}_{G / H}^{l-1}(\mathcal{E}) \rightarrow 0 \\
& \text { with G-linearization. }
\end{aligned}
$$

Proof. By literature of Maakestad [4] sequence 2.2.1 is an exact sequence of locally free $\mathcal{O}_{G / H}$-modules. The scheme $Y$ is equipped with the diagonal $G$-action. It follows $p_{*}$ and $q^{*}$ preserve $G$-linearizations. We get a diagram of exact sequences of $\mathcal{O}_{Y}$-modules with a $G$-linearization

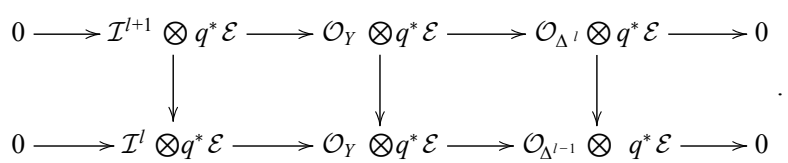

Since $p_{*}$ preserves $G$-linearization we get a morphism

$\phi: \mathcal{P}_{G / H}^{l}(\mathcal{E}) \rightarrow \mathcal{P}_{G / H}^{l-1}(\mathcal{E})$

preserving the $G$-linearization, and the Proposition is proved.

Let $\mathrm{g}=\operatorname{Lie}(G)$ and $\mathrm{h}=\operatorname{Lie}(H)$. Let $H_{l} \subseteq H$ be a Levi subgroup of $H$. It follows $H_{l}$ is a maximal linearly reductive subgroup of $H$. The group $H_{l}$ is not unique but all such groups are conjugate under automorphisms of $H$. Let $x \in G$ / $H$ be the $K$-rational point defined by the identity $e \in G$.

Theorem 2.3. There is for all $l \geq 1$ an isomorphism

$$
\mathcal{P}_{X}^{l}(\mathcal{E})(x)^{*} \cong \mathcal{E}(x)^{*} \otimes\left(\oplus_{i=}^{l} \operatorname{Sym}^{i}(\mathfrak{g} / \mathfrak{h})\right)
$$

of L-modules.

Proof. Dualize the sequence 2.2.1 and take the fiber at $x$ to get the exact sequence

$$
0 \rightarrow \mathcal{P}_{X}^{l-1}(\mathcal{E})(x)^{*} \rightarrow \mathcal{P}_{X}^{l}(\mathcal{E})(x)^{*} \rightarrow \mathcal{E}(x)^{*} \otimes \operatorname{Sym}^{l}(\mathfrak{g} / \mathfrak{h}) \rightarrow 0
$$

of $H$-modules (and $H_{l}$-modules). This sequence splits since $H_{l}$ is linearly reductive and the Theorem follows by induction on $l$.

Hence the study $\mathcal{P}_{X}^{l}(\mathcal{E})(x)^{*}$ as $H_{l}$-module is reduced to the study of $\mathcal{E}(x)^{*}$ and $\operatorname{Sym}^{l}(\mathrm{~g} / \mathrm{h})$.

Let $W \subseteq V$ be $K$-vector spaces of dimension $m$ and $m+n$ and let $G$ $=\mathrm{SL}(V)$ and $P \subseteq G$ the subgroup fixing $W$. It follows $G / P=\mathbb{G}(m, m+$ $n)$ is the grassmannian of $m$-planes in $V$. Let $\mathrm{g}=\operatorname{Lie}(G)$ and $\mathrm{p}=\operatorname{Lie}(P)$. Fix a basis $e_{1}, . ., e_{m}$ for $W$ and $e_{1}, . ., e_{m}, e_{m+1}, . ., e_{m+n}$ for $V$. It follows the $K$-rational points of $P$ are matrices $M$ on the form

$$
M=\left(\begin{array}{cc}
A & X \\
0 & B
\end{array}\right)
$$

where $\operatorname{det}(A) \operatorname{det}(B)=1, A$ an $m \times m$-matrix and $B$ an $n \times n$-matrix. Let $P_{l} \subseteq P$ be the subgroup defined as follows: The $K$-rational points of $P_{l}$ are matrices $M$ on the form

$$
M=\left(\begin{array}{cc}
A & 0 \\
0 & B
\end{array}\right)
$$

where $\operatorname{det}(A) \operatorname{det}(B)=1$ and similarly $A$ an $m \times m$-matrix and $B$ an $n$ $\times n$-matrix. It follows $P_{l}$ is a Levi subgroup of $P$, hence it is a maximal linearly reductive subgroup.

Proposition 2.4. There is a canonical isomorphism

$\mathfrak{g} / \mathfrak{p} \cong \operatorname{Hom}(W, V / W)$ 
of P-modules.

Proof. By definition $\mathrm{g}=\mathrm{sl}(V)$, hence $\varphi \in \mathrm{g}$ is a map

$\varphi: V \rightarrow V$

with $\operatorname{tr}(\varphi)=0$. Let $i: W \rightarrow V$ be the inclusion map and $p: V \rightarrow V / W$ the projection map. Define the following map:

$\mathrm{J}^{\prime}: \mathfrak{g} \rightarrow \operatorname{Hom}(W, V / W)$

by

$j^{\prime}(\varphi)=p \circ \varphi i$.

It follows $j(\mathrm{p})=0$ hence we get a well defined map

$j: \mathrm{g} / \mathrm{p} \rightarrow \operatorname{Hom}(W, V / W)$

defined by

$j(\bar{\phi})=p \circ \phi \circ i$.

One checks $\mathrm{g} / \mathrm{p}$ and $\operatorname{Hom}(W, V / W)$ are $P$-modules and $j$ a morphism of $P$-modules. It is an isomorphism and the Proposition follows.

Corollary 2.5. On $X=\mathbb{G}(m, m+n)$ there is an isomorphism

$\mathcal{P}_{X}^{l}(\mathcal{E})(x)^{*} \cong \mathcal{E}(x)^{*} \otimes\left(\oplus_{i=0}^{l} \operatorname{Sym}^{i}(\operatorname{Hom}(W, V / W))\right.$

of $P_{l}$-modules.

Proof. The proof follows from Theorem 2.3 and Proposition 2.4.

There is an isomorphism of $P$-modules

$\operatorname{Hom}(W, V / W) \cong W^{*} \otimes V / W$

hence the decomposition into irreducible components of the module $\operatorname{Sym}^{i}\left(\mathrm{~W}^{*} \otimes V / W\right)$ as $P_{l}$-module may be done using the Cauchy formula (Appendix B).

Let $\lambda-|i|$ denote $\lambda$ is a partition of the integer $i$ If $\lambda=\left\{\lambda_{1}, . ., \lambda_{d}\right\}$ is a partition of an integer $l$, let $\mu(\lambda)$ denote the following partition:

$$
\mu(\lambda)_{i}=1-\lambda_{d+1-i}
$$

Let for any partition $\lambda$ of an integer $l$ and any vector space $W, \mathbb{S}_{\lambda}(W)$ denote the Schur-Weyl module of $\lambda$.

Corollary 2.6. There is an isomorphism

$$
\mathcal{P}_{X}^{l}(\mathcal{E})(x)^{*} \cong \mathcal{E}(x)^{*} \otimes\left(\oplus_{i=0}^{l}\left(\bigoplus_{\lambda-i \mid} \mathbb{S}_{\lambda}\left(W^{*}\right) \otimes \mathbb{S}_{\mu(\lambda)}(V / W)\right)\right)
$$

of $\operatorname{SL}(W) \times \operatorname{SL}(V / W)$-modules.

Proof. By Corollary 2.5 there is an isomorphism

$\mathcal{P}_{X}^{l}(\mathcal{E})(x)^{*} \cong \mathcal{E}(x)^{*} \otimes\left(\oplus_{i=0}^{l} \operatorname{Sym}^{i}(\operatorname{Hom}(W, V / W))\right.$

of $P_{l}$-modules and $\operatorname{SL}(W) \times \operatorname{SL}(V / W)$-modules, since $\operatorname{SL}(W) \times$ $\mathrm{SL}(V / W) \subseteq P_{l}$ is a closed subgroup. Since

$\operatorname{Sym}^{i}(\operatorname{Hom}(W, V / W)) \cong \operatorname{Sym}^{i}\left(W^{*} \otimes V / W\right)$

the result follows from the Cauchy formula (Appendix B or [14]).

Example 2.7. Calculation of the cohomology group $\mathrm{H}^{i}\left(X, \wedge^{j} \mathcal{P}_{X}^{l}\left(\mathcal{O}_{X}(d)\right)^{*}\right)$.

In the following we use the notation introduced in litertature of Jantzen [13]. Let $P_{\text {semi }}=\mathrm{SL}(m) \times \mathrm{SL}(n) \subseteq P$ be the semi simplification of $P$. We get a vector bundle

$\pi: G / P_{\text {semi }} \rightarrow G / P=\mathbb{G}(m, m+n)$.
Let $X=G / P$ and $Y=G / P_{\text {semi }}$ Given any finite dimensional $P$-module $W$, let $\mathcal{L}_{X}(W)$ denote its corresponding $\mathcal{O}_{X}$-module. Let $W_{\text {semi }}$ denote the restriction of $W$ to $P_{\text {semi }}$. By the results of Perkinson [13] it follows there is an isomorphism

$$
\pi^{*} \mathcal{L}_{X}(W) \cong \mathcal{L}_{Y}\left(W_{\text {semi }}\right)
$$

of locally free sheaves. This will help calculating the higher cohomology group

$\mathrm{H}^{i}\left(X, \mathcal{L}_{X}(W)\right)$

since $P_{\text {semi }}$ is semi simple and $\pi$ is a locally trivial fibration. If $W$ is the $P$-module corresponding to the dual of the $j$ th exterior power of the jet bundle $\wedge^{j} \mathcal{P}_{X}^{l}\left(\mathcal{O}_{X}(d)\right)^{*}$ we can use this construction to calculate the cohomology group

$\mathrm{H}^{i}\left(X, \wedge^{j} \mathcal{P}_{X}^{l}\left(\mathcal{O}_{X}(d)\right)^{*}\right)$.

Such a calculation will be by the results of Maakestad [11], Example 5.12 give information on resolutions of the ideal sheaf of $D^{l}\left(\mathcal{O}_{X}(d)\right)$ since the push down of the Koszul complex 1.0.1 is the locally trivial sheaf

$$
\mathcal{O}(-j) \otimes \mathrm{H}^{i}\left(X, \wedge^{j} \mathcal{P}_{X}^{l}\left(\mathcal{O}_{X}(d)\right)^{*}\right) \text {. }
$$

To describe the locally trivial sheaf $\mathcal{O}(-j) \otimes \mathrm{H}^{i}\left(X, \wedge^{j} \mathcal{P}_{X}^{l}\left(\mathcal{O}_{X}(d)\right)^{*}\right)$ for all $i, j$ we need to calculate the dimension $h^{i}\left(X, \wedge^{j} \mathcal{P}_{X}^{l}\left(\mathcal{O}_{X}(d)\right)^{*}\right)$ and this calculation may be done using the approach indicated above.

Let $m=2, n=4$ and $X=\mathbb{G}(2,4)$.

Corollary 2.8. There is an isomorphism

$\mathcal{P}_{X}^{l}(\mathcal{E})(x)^{*} \cong \mathcal{E}(x)^{*} \otimes\left(\oplus_{i=0}^{l} \oplus_{j=0}^{n} \operatorname{Sym}^{2 j+m}\left(W^{*}\right) \otimes \operatorname{Sym}^{2 j+m}(V / W)\right)$ of SL(2) $\times \operatorname{SL}(2)$-modules. Here $(n, m)=\left(\frac{i}{2}, 0\right)$ if $i=2 n$ and $\left(\frac{i-1}{2}, 1\right)$ if $i=2 n+1$.

Proof. This follows from Corollary 2.5 and Proposition 5.1 .

\section{On Canonical Filtrations and Jet Bundles on Projective Space}

In this section we study the canonical filtration for the dual of the $\operatorname{SL}(V)$-module of global sections of an invertible sheaf on the grassmannian. We classify the canonical filtration on projective space and as a result recover known formulas on the splitting type of the jet bundle as abstract locally free sheaf.

Let $W \subseteq V$ be vector spaces over $K$ of dimension $m$ and $m+n$. Let $W$ have basis $e_{1}, \ldots, e_{m}$ and $V$ have basis $e_{1}, \ldots, e_{m+n}$. Let $V^{*}$ have basis $x_{1}$, .., $x_{m+n}$. Let $G=\mathrm{SL}(V)$ and $P \subseteq G$ the parabolic subgroup of elements fixing $W$. It follows there is a quotient morphism

$$
\pi: G \rightarrow G / P
$$

and $G / P \cong \mathbb{G}(m, m+n)$ is the grassmannian of $m$-planes in $V$. Let $\mathbb{P}=\mathbb{G}(1, n+1)=\mathbb{P}\left(V^{*}\right)$. Let $L^{d}=\operatorname{Sym}^{d}\left(\wedge^{m} W\right)$. There is an inclusion of $P$-modules $L^{d} \subseteq \operatorname{Sym}^{d}\left(\wedge^{m} V\right)$. Since $K$ has characteristic zero there is an inclusion of $G$-modules

$$
\mathrm{H}^{0}\left(G / P, \mathcal{O}_{G / P}(d)\right)^{*} \subseteq \operatorname{Sym}^{d}\left(\wedge^{m} V^{*}\right)^{*} \cong \operatorname{Sym}^{d}\left(\wedge^{m} V\right) .
$$

Let $\mathrm{g}=\operatorname{Lie}(G)$ and $\mathrm{p}=\operatorname{Lie}(P)$. Let $\mathrm{U}(\mathrm{g})$ be the universal enveloping algebra og g and let $\mathrm{U}_{l}(\mathrm{~g})$ be the $l$ 'th term to its canonical filtration.

By the Corollary 3.11 in studies of Maakestad [15] there is for all 1 $\leq l \leq d$ an exact sequence of $P$-modules

$0 \rightarrow \mathcal{P}_{\mathbb{G}}^{l}\left(\mathcal{O}_{\mathbb{G}}(d)\right)(x)^{*} \rightarrow \mathrm{H}^{0}\left(\mathbb{G}, \mathcal{O}_{\mathbb{G}}(d)\right)^{*} \rightarrow \mathrm{H}^{0}\left(\mathbb{G}, \mathfrak{m}^{l+1} \mathcal{O}_{\mathbb{G}}(d)\right)^{*} \rightarrow 0$. 
Since the grassmannian is projectively normal in the Plucker embedding we get an inclusion

$$
\mathrm{H}^{0}\left(\mathbb{G}, \mathcal{O}_{\mathbb{G}}(d)\right)^{*} \subseteq \operatorname{Sym}^{d}\left(\wedge^{m} V\right)
$$

of $P$-modules. The highest weight vector for $\mathrm{H}^{0}\left(\mathbb{G}, \mathcal{O}_{\mathbb{G}}(d)\right)^{*}$ is the line $L^{d}$ $=\operatorname{Sym}^{d}\left(\wedge^{m} W\right)$. Let $\operatorname{ann}\left(L^{d}\right) \subseteq \mathrm{U}(\mathfrak{g})$ be the left annihilator ideal of $L^{d}$. It is the ideal generated by elements $x \in \mathrm{U}(\mathfrak{g})$ with the property $x\left(L^{d}\right)=$ 0 . Let $a n n_{l}\left(L^{d}\right)$ be its canonical filtration. We get an exact sequence of $G$-modules

$$
0 \rightarrow \operatorname{ann}\left(L^{d}\right) \otimes L^{d} \rightarrow \mathrm{U}(\mathfrak{g}) \otimes L^{d} \rightarrow \mathrm{H}^{0}\left(X, \mathcal{O}_{X}(d)\right)^{*} \rightarrow 0
$$

and an exact sequence of $P$-modules

$$
0 \rightarrow \operatorname{ann}_{l}\left(L^{d}\right) \otimes L^{d} \rightarrow \mathrm{U}_{l}(\mathfrak{g}) \otimes L^{d} \rightarrow \mathrm{U}_{l}(\mathfrak{g}) L^{d} \rightarrow 0
$$

for all $l \geq 1$. The $G$-module $\mathrm{U}(\mathfrak{g}) \otimes L^{d}$ is the generalized Verma module corresponding to the $P$-module defined by $L^{d}=\operatorname{Sym}^{d}\left(\wedge^{m} V\right)$. There is an inclusion of $P$-modules

$$
\mathrm{U}_{l}(\mathfrak{g}) L^{d} \subseteq \mathrm{H}^{0}\left(\mathbb{G}, \mathcal{O}_{\mathbb{G}}(d)\right)^{*} .
$$

Definition 3.1. Let $\left\{\mathrm{U}_{l}(\mathfrak{g}) L^{d}\right\}_{l \geq 1}$ be the canonical filtration for $\mathrm{H}^{0}(\mathbb{G}, \mathcal{O} \mathbb{G}(d))^{*}$.

Lemma 3.2. Assume $y \in \mathfrak{g}$ and $x_{1} \cdots x_{i} \in \mathrm{U}_{i}(\mathfrak{g})$ with $x_{i} \in \mathfrak{g}$. The following holds:

$$
y\left(x_{1} \cdots x_{i}\right)=\left(x_{1} \cdots x_{i}\right) y+w
$$

where $w \in \mathrm{U}_{i-1}(\mathfrak{g}) \omega \in U_{i-1}(\mathfrak{g})$.

Proof. The proof is by induction.

The Lie algebra $p$ is the sub Lie algebra of $\mathfrak{g}=\mathrm{sl}(V)$ given by matrices $M$ of the following type:

$$
M=\left(\begin{array}{cc}
A & X \\
0 & B
\end{array}\right)
$$

where $A$ is an $m \times m$-matrix, $B$ and $n \times n$-matrix and $\operatorname{tr}(A)+\operatorname{tr}(B)=$ 0 . Let $\mathrm{p}_{L}$ be the sub Lie algebra of $\mathfrak{p}$ consisting of matrices $M \in \mathfrak{p}$ of the following type:

$$
M=\left(\begin{array}{cc}
A & X \\
0 & B
\end{array}\right)
$$

where $\operatorname{tr}(A)+\operatorname{tr}(B)=0$.

\section{Proposition 3.3.}

The sub Lie algebra $\mathfrak{p}_{L} \subseteq \mathfrak{p}$ is a sub P-module of $\mathfrak{p}$.

There is an exact sequence of $P$-modules

$0 \rightarrow \mathfrak{p} / \mathfrak{p}_{L} \rightarrow \mathfrak{g} / \mathfrak{p}_{L} \rightarrow \mathfrak{g} / \mathfrak{p} \rightarrow 0$.

and $\mathfrak{p} / \mathfrak{p}_{L}$ is the trivial $P$-module.

The following holds:

$$
\operatorname{dim}_{K}\left(L^{d-k} \otimes \operatorname{Sym}^{k}\left(\mathfrak{g} / \mathfrak{p}_{L} \otimes L\right)\right)=\left(\begin{array}{c}
m n+k \\
m n
\end{array}\right) .
$$

There is a filtration of P-modules

$$
\begin{aligned}
& 0=G_{l+1} \subseteq G_{l} \subseteq \cdots \subseteq G_{0}=L^{d-l} \otimes \operatorname{Sym}^{l}\left(\mathfrak{g} / \mathfrak{p}_{L} \otimes L\right) \\
& \text { with quotients } \\
& G_{i} / G_{i+1} \cong L^{d-(l-i)} \otimes \operatorname{Sym}^{l-i}((\mathfrak{g} / \mathfrak{p} \otimes L) \\
& \text { for } 1 \leq i \leq k
\end{aligned}
$$

Assume $\operatorname{dim}_{k}(W)=1$ and let $W=L$. There is an exact sequence of

\section{P-modules}

$$
0 \rightarrow \mathfrak{p}_{L} \otimes L \rightarrow \mathfrak{g} \otimes L \rightarrow V \rightarrow 0
$$

giving an isomorphism of $P$-modules $\mathfrak{g} / \mathfrak{p}_{L} \otimes L \cong V$.

Proof. We prove 3.3.1: In the following $A, a$ are square matrices of size $m$ and $b, B$ square matrices of size $n$. The $K$-rational points of the group $P$ are matrices $g$ on the form

$$
g=\left(\begin{array}{ll}
A & X \\
0 & B
\end{array}\right)
$$

where $\operatorname{det}(A) \operatorname{det}(B)=1$. Assume $x \in \mathrm{p}$ is the following element:

$$
x=\left(\begin{array}{ll}
a & x \\
0 & b
\end{array}\right)
$$

with $\operatorname{tr}(a)+\operatorname{tr}(b)=0$. It follows $g(x)=g x g^{-1}$ has $\operatorname{tr}\left(g x g^{-1}\right)=\operatorname{tr}\left(g g^{-1} x\right)=$ $\operatorname{tr}(x)=0$ hence $g x g^{-1} \in \mathfrak{p}$ and $\mathfrak{p}$ is a $P$-module. Assume $x \in \mathfrak{p}_{L}$ ie $\operatorname{tr}(a)=$ $\operatorname{tr}(b)=0$. It follows

$$
g x g^{-1}=\left(\begin{array}{cc}
a A a^{-1} & * \\
0 & b B b^{-1}
\end{array}\right)
$$

and $\operatorname{tr}\left(a A a^{-1}\right)+\operatorname{tr}\left(a a^{-1} A\right)=\operatorname{tr}(A)=0$ hence $g(x) \in \mathfrak{p}_{L}$ and 3.3.1 is proved.

We prove 3.3.2: By 3.3.1 it follows $\mathfrak{p}_{L} \subseteq \mathfrak{p}$ is a sub $P$-module. One checks $\mathfrak{p} / \mathfrak{p}_{L}$ is a trivial $P$-module. We clearly get an exact sequence of $P$-modules and 3.3.2 is proved.

We prove 3.3.3: Since

$\operatorname{dim}_{K}(\mathfrak{g})=(m+n)^{2}-1=n^{2}+2 m n+m^{2}-1$

and

$\operatorname{dim}_{K}\left(\mathfrak{p}_{L}\right)=m^{2}+m n+n^{2}-2$

it follows $\operatorname{dim}_{k}\left(\mathfrak{g} / \mathfrak{p}_{L}\right)=m n+1$. It follows

$$
\operatorname{dim}_{K}\left(L^{d-l} \otimes \operatorname{Sym}^{l}\left(\mathfrak{g} / \mathfrak{p}_{L} \otimes l\right)=\left(\begin{array}{c}
m n+1+l-1 \\
m n+1-1
\end{array}\right)=\left(\begin{array}{c}
m n+l \\
m n
\end{array}\right) .\right.
$$

We prove 3.3.4: Since $\mathfrak{p} / \mathfrak{p}_{L}$ is a trivial $P$-module there are isomorphisms of $P$-modules

$$
\begin{aligned}
& L^{d-(k-i)} \otimes \operatorname{Sym}^{k-i}\left(\mathfrak{g} / \mathfrak{p}_{L} \otimes L\right) \cong L^{d-k} \otimes L^{i} \otimes \operatorname{Sym}^{k-i}\left(\mathfrak{g} / \mathfrak{p}_{L} \otimes L\right) \cong \\
& L^{d-k} \otimes \operatorname{Sym}^{i}\left(\mathfrak{p} / \mathfrak{p}_{L} \otimes L\right) \otimes \operatorname{Sym}^{k-i}\left(\mathfrak{g} / \mathfrak{p}_{L} \otimes L\right)
\end{aligned}
$$

for all $1 \leq i \leq k$. We get an injection

$j: L^{d-k} \otimes \operatorname{Sym}^{i}\left(\mathfrak{p} / \mathfrak{p}_{L} \otimes L\right) \otimes \operatorname{Sym}^{k-i}\left(\mathfrak{g} / \mathfrak{p}_{L} \otimes L\right) \rightarrow L^{d-k} \otimes \operatorname{Sym}^{k}\left(\mathfrak{g} / \mathfrak{p}_{L} \otimes L\right)$ defined by

$j\left(L^{d-k} \otimes \overline{y_{1}} \otimes L \cdots \overline{y_{i}} \otimes L \otimes \overline{x_{1}} \otimes L \cdots \overline{x_{k-i}} \otimes L\right)=L^{d-k} \otimes \overline{y_{1}} \otimes L \cdots \overline{y_{i}} \otimes L \overline{x_{1}} \otimes L \cdots \overline{x_{k-i}}$.

The injection $j$ gives rise to an injection

$L^{d-(k-i)} \otimes \operatorname{Sym}^{k-i}\left(\mathfrak{g} / \mathfrak{p}_{L} \otimes L\right) \cong L^{d-k} \otimes \operatorname{Sym}^{i}\left(\mathfrak{p} / \mathfrak{p}_{L} \otimes L\right) \otimes \operatorname{Sym}^{k-i}\left(\mathfrak{g} / \mathfrak{p}_{L} \otimes L\right) \rightarrow{ }^{j}$

$L^{d-k} \otimes \operatorname{Sym}^{k}\left(\mathfrak{g} / \mathfrak{p}_{L} \otimes L\right)$

of $P$-modules for all $1 \leq i \leq k$. The exact sequence

$0 \rightarrow \mathfrak{p} / \mathfrak{p}_{L} \rightarrow \mathrm{g} / \mathfrak{p}_{L} \rightarrow \mathrm{g} / \mathfrak{p} \rightarrow 0$

gives rise to a filtration of $P$-modules

$0=F_{l+1} \subseteq F_{l} \subseteq \cdots \subseteq F_{0}=\operatorname{Sym}^{l}\left(\mathfrak{g} / \mathfrak{p}_{L} \otimes L\right)$

with quotients

$F_{i} / F_{i+1} \cong L^{i} \otimes \operatorname{Sym}^{l-i}(\mathfrak{g} / \mathfrak{p} \otimes L)$. 
Put $G_{i}=L^{d-1} \otimes F_{i}$. It follows

$G_{i}=L^{d-(l-i)} \otimes \operatorname{Sym}^{l-i}\left(\mathfrak{g} / \mathfrak{p}_{L} \otimes L\right)$.

There is an isomorphism

$G_{i} / G_{i+1} \cong L^{d-(l-i)} \otimes \operatorname{Sym}^{l-i}(\mathfrak{g} / \mathfrak{p} \otimes L)$

and claim 3.3.4 is proved.

We prove 3.3.5: Let $V=K\left\{e_{0}, . ., e_{n}\right\}$ and $L=W=e_{0}$. It follows $P \subseteq G$ $=\mathrm{SL}(V)$ is the group whose $K$-rational points are the following:

$$
g=\left(\begin{array}{ll}
a & * \\
0 & B
\end{array}\right)
$$

with $a=\frac{1}{\operatorname{det}(B)}$. Also $B$ is an $n \times n$-matrix with coefficients in $K$. By definition the maps in the sequence are maps of $P$-modules. It follows $\mathrm{p}=\operatorname{Lie}(P)$ is the Lie algebra whose elements $x$ are matrices on the following form:

$$
x=\left(\begin{array}{cc}
-\operatorname{tr}(B) & * \\
0 & B
\end{array}\right)
$$

where $B$ is any $n \times n$-matrix with coefficients in $K$. The sub Lie algebra $\mathfrak{p}_{L} \subseteq \mathfrak{p}$ is the Lie algebra of matrixes $x \in \mathfrak{p}$ on the following form:

$$
x=\left(\begin{array}{ll}
0 & * \\
0 & B
\end{array}\right)
$$

where $B$ is any $n \times n$-matrix with $\operatorname{tr}(B)=0$. Let $x_{i} \in \mathfrak{g}$ be the following element: Let the first column vector of $x_{i}$ be the vector $e_{i}$ and let the rest of the entries be such that $\operatorname{tr}\left(x_{i}\right)=0$. It follows $x_{i} \otimes e_{0} \in \mathfrak{g} \otimes L$ and $x_{i}\left(e_{0}\right)$ $=e_{i}$ hence the vertical map is surjective. One easily checks the sequence is exact and 3.3.5 is proved.

We get two $P$-modules: $\mathfrak{p}_{L} \subseteq \mathfrak{p}$ and $L^{i}=\operatorname{Sym}^{i}\left(\wedge^{m} W\right) \subseteq \operatorname{Sym}^{i}\left(\wedge^{m} V\right)$. We get for all $1 \leq k \leq d$ a $P$-module

$$
L^{d-k} \otimes \operatorname{Sym}^{k}\left(\mathfrak{g} / \mathfrak{p}_{L} \otimes L\right) .
$$

There is an injection of $P$-modules

$$
i: L^{d-k} \otimes \operatorname{Sym}^{k}\left(\mathfrak{g} / \mathfrak{p}_{L} \otimes L\right) \rightarrow \operatorname{Sym}^{d}\left(\wedge^{m} V\right)
$$

defined by

$$
i\left(L^{d-k} \otimes \overline{x_{1}} \otimes L \cdots \overline{x_{k}} \otimes L\right)=L^{d-k} x_{1}(L) \cdots x_{k}(L) .
$$

There are natural embeddings of $P$-modules

$$
\begin{aligned}
& U_{k}(\mathfrak{g}) L^{d} \subseteq \operatorname{Sym}^{d}\left(\wedge^{m} V\right) \\
& \text { and } \\
& L^{d-(k-1)} \otimes \operatorname{Sym}^{k-1}\left(\mathfrak{g} / \mathfrak{p}_{L} \otimes L\right) \subseteq L^{d-k} \otimes \operatorname{Sym}^{k}\left(\mathfrak{g} / \mathfrak{p}_{L} \otimes L\right) \subseteq \operatorname{Sym}^{d}\left(\wedge^{m} V\right) .
\end{aligned}
$$

Assume in the following $m=1$ and $L=W$. It follows $\mathbb{G}=\mathcal{P}\left(V^{*}\right)=$ $\mathcal{P}$ is projective $n$-space.

Proposition 3.4. Let $x_{1} \cdots x_{k}\left(L^{d}\right) \in U_{k}(\mathfrak{g}) L^{d}$. The following formula holds:

$$
\begin{aligned}
& x_{1} \cdots x_{k}\left(L^{d}\right)=\alpha L^{d-k} x_{1}(L) \cdots x_{k}(L)+\omega \\
& \text { where } \omega \in L^{d-(k-1)} \otimes \operatorname{Sym}^{k-1}\left(\mathfrak{g} / \mathfrak{p}_{L} \otimes L\right) .
\end{aligned}
$$

Proof. we prove the result by induction on $k$. Assume $k=1$ and let $x\left(L^{d}\right) \in \mathrm{U}_{1}(\mathfrak{g}) L^{d}$. It follows $x\left(L^{d}\right)=d L^{d-1} x(L) \in L^{d-1} \otimes \operatorname{Sym}^{1}\left(\mathfrak{g} / \mathfrak{p}_{L} \otimes L\right)$ and the claim holds for $k=1$. Assume the result is true for $k$. Hence

$$
x_{1} \cdots x_{k}\left(L^{d}\right)=\alpha L^{d-k} x_{1}(L) \cdots x_{k}(L)+\omega
$$

with $\omega \in L^{d-(k-1)} \otimes \operatorname{Sym}^{k-1}\left(\mathfrak{g} / \mathfrak{p}_{L} \otimes L\right)$. Assume

$\omega=\sum_{i} \alpha_{i} L^{d-(k-1)} x_{1}^{i}(L) \cdots x_{k-1}^{i}(L)$.

We get

$$
\begin{aligned}
& x_{0} x_{1} \cdots x_{k}\left(L^{d}\right)=x_{0}\left(\alpha L^{d-k} x_{1}(L) \cdots x_{k}(L)+\omega\right)= \\
& \alpha(d-k) L^{d-(k+1)} x_{0}(L) x_{1}(L) \cdots x_{k}(L)+ \\
& \sum_{j} \alpha L^{d-k} x_{1}(L) \cdots x_{0}\left(x_{j}(L)\right) \cdots x_{k}(L)+ \\
& \sum_{i} \alpha_{i}(d-(k-1)) L^{d-k} x_{0}(L) x_{1}^{i}(L) \cdots x_{k-1}^{i}(L)+ \\
& \sum_{i} \sum_{l} \alpha_{i} L^{d-(k-1)} x_{1}^{i}(L) \cdots x_{0}\left(x_{l}^{i}(L)\right) \cdots x_{k-1}^{i}(L) .
\end{aligned}
$$

Let $z_{j}(L)=x_{0}\left(x_{j}(L)\right)$ and $z_{l}^{i}(L)=x\left(x_{l}^{i}(L)\right)$. Such elements exist since $\mathfrak{g} / \mathfrak{p}_{L} \otimes L \cong V$ as $P$-module. Let

$$
\begin{aligned}
& \omega=\sum_{j} \alpha L^{d-k} x_{1}(L) \cdots z_{j}(L) \cdots x_{k}(L)+ \\
& \sum_{i} \alpha_{i}(d-(k-1)) L^{d-k} x_{0}(L) \cdots x_{1}^{i}(L) \cdots x_{k-1}^{i}(L)+ \\
& \sum_{i} \sum_{l} \alpha_{i} L^{d-(k-1)} x_{1}^{i}(L) \cdots z_{l}^{i}(L) \cdots x_{k-1}^{i}(L) .
\end{aligned}
$$

it follows $\omega \in L^{d-k} \otimes \operatorname{Sym}^{k}\left(\mathfrak{g} / \mathfrak{p}_{L} \otimes L\right)$. Moreover

$x_{0} x_{1} \cdots x_{k}\left(L^{d}\right)=\tilde{\alpha} L^{d-(k+1)} x_{0}(L) \cdots x_{k}(L)+\omega$

where $\tilde{\alpha}=(d-k) \alpha$. The Proposition is proved.

Theorem 3.5. There is for all $1 \leq l \leq d$ an isomorphism

$\mathrm{U}_{l}(\mathfrak{g}) L^{d} \cong L^{d^{l}} \otimes \operatorname{Sym}^{l}\left(\mathfrak{g} / \mathfrak{p}_{L} \otimes L\right)$

of P-modules.

Proof. There are embeddings of $P$-modules

$\mathrm{U}_{l}(\mathfrak{g}) L^{d} \subseteq \operatorname{Sym}^{d}(V)$

and

$L^{d-l} \otimes \operatorname{Sym}^{l}\left(\mathfrak{g} / \mathfrak{p}_{L} \otimes L\right) \subseteq \operatorname{Sym}^{d}(V)$.

Recall fromstudies of Maakestad [1] itfollows $\operatorname{dim}_{K}\left(\mathrm{U}_{l}(\mathfrak{g}) L^{d}\right)=\left(\begin{array}{c}l+n \\ n\end{array}\right)$ where $\operatorname{dim}_{k}(V)=n+1$. Assume $z=x_{1} \cdots x_{l}\left(L^{d}\right) \in \mathrm{U}_{l}(\mathfrak{g}) L^{d}$. It follows from Proposition 3.4

$z=\alpha L^{d-l} x_{1}(L) \cdots x_{l}(L)+\omega$

where

$$
\in L^{d-(l-1)} \operatorname{Sym}^{l-1}\left(\mathfrak{g} / \mathfrak{p}_{L} \otimes L\right) \subseteq L^{d-l} \otimes \operatorname{Sym}^{l}\left(\mathfrak{g} / \mathfrak{p}_{L} \otimes L\right) .
$$

Since

$\alpha L^{d-l} x_{1}(L) \cdots x_{l}(L) \in L^{d-l} \otimes \operatorname{Sym}^{l}\left(\mathfrak{g} / \mathfrak{p}_{L} \otimes L\right)$

it follows $z \in L^{d-l} \otimes \operatorname{Sym}^{l}\left(\mathfrak{g} / \mathfrak{p}_{L} \otimes L\right)$ Hence we get an inclusion of $P$-modules $\mathrm{U}_{l}(\mathfrak{g}) L^{d} \subseteq L^{d-l} \otimes \operatorname{Sym}^{l}\left(\mathfrak{g} / \mathfrak{p}_{L} \otimes L\right)$.

Since

$\operatorname{dim}_{K}\left(\mathrm{U}_{l}(\mathfrak{g}) L^{d}\right)=\operatorname{dim}_{K}\left(L^{d-l} \otimes \operatorname{Sym}^{l}\left(\mathfrak{g} / \mathfrak{p}_{L} \otimes L\right)\right)$

the Theorem follows.

Corollary 3.6. There is for all $1 \leq l \leq d$ an isomorphism

$\mathcal{P}_{\mathbb{P}}^{l}\left(\mathcal{O}_{\mathbb{P}}(d)\right)(x) \cong\left(L^{*}\right)^{d-l} \otimes \operatorname{Sym}^{1}\left(V^{*}\right)$

of P-modules. 
Proof. There is by studies of Maakestad [1], Theorem 3.10 an isomorphism

$$
\mathcal{P}_{\mathbb{P}}^{l}\left(\mathcal{O}_{\mathbb{P}}(d)\right)(x)^{*} \cong \mathrm{U}_{l}(\mathfrak{g}) L^{d}
$$

of $P$-modules. From this isomorphism and Theorem 3.5 the Corollary follows since

$$
\begin{aligned}
& \left(L^{d-l} \otimes \operatorname{Sym}^{l}\left(\mathfrak{g} / \mathfrak{p}_{L} \otimes L\right)\right)^{*} \cong\left(L^{*}\right)^{d-l} \otimes \operatorname{Sym}^{l}\left(V^{*}\right) \\
& \text { as } P \text {-modules. }
\end{aligned}
$$

Note: Corollary 3.6 is proved in literature of Maakestad [5] Theorem 2.4 using more elementary techniques.

Let $Y=\operatorname{Spec}(K)$ and $\pi: \mathcal{P}\left(V^{*}\right) \rightarrow Y$ be the structure morphism. Let $\mathcal{P}=\mathcal{P}\left(V^{*}\right)$. Since $\operatorname{Sym}^{1}\left(V^{*}\right)$ is a finite dimensional $\operatorname{SL}(V)$-module it follows it is a free $\mathcal{O}_{Y}$-module with an $\operatorname{SL}(V)$-linearization. It follows $\pi^{*} \operatorname{Sym}^{1}\left(V^{*}\right)$ is a locally free $\mathcal{O P}$-module with an $\operatorname{SL}(V)$-linearization since $\pi^{*}$ preserves the $\mathrm{SL}(V)$-linearization.

Proposition 3.7. There is for all $1 \leq l \leq d$ an isomorphism

$$
\mathcal{P}_{\mathbb{P}}^{l}\left(\mathcal{O}_{\mathbb{P}}(d)\right) \cong \mathcal{O}_{\mathbb{P}}(d-l) \otimes \pi^{*} \operatorname{Sym}^{l}\left(V^{*}\right)
$$

of locally free $\mathcal{O P}$-modules with an $\mathrm{SL}(V)$-linearization.

Proof. Let $P \subseteq \operatorname{SL}(V)$ be the subgroup fixing the line $L \in V$ There is an exact equivalence of categories

$$
\underline{\bmod }(P) \cong \underline{\bmod }^{G}\left(\mathcal{O}_{G / P}\right) \text {. }
$$

The $P$-module corresponding to $\mathcal{O}_{\mathbb{P}}(d-l) \otimes \pi^{*} \operatorname{Sym}^{l}\left(V^{*}\right) \quad$ is $(L)^{d-l} \otimes \operatorname{Sym}^{l}\left(V^{*}\right)$. By the equivalence 3.7.1 and Corollary 3.6 we get an isomorphism

$$
\mathcal{P}_{\mathbb{P}}^{l}\left(\mathcal{O}_{\mathbb{P}}(d)\right) \cong \mathcal{O}_{\mathbb{P}}(d-l) \otimes \pi^{*} \operatorname{Sym}^{l}\left(V^{*}\right)
$$

of locally free sheaves with $\operatorname{SL}(V)$-linearization and the Proposition is proved.

We get a formula for the splitting type of $\mathcal{P}_{\mathbb{P}}^{l}\left(\mathcal{O}_{\mathbb{P}}(d)\right)$ on projective space:

Corollary 3.8. There is for all $1 \leq l \leq d$ an isomorphism

$\mathcal{P}_{\mathbb{P}}^{l}(\mathcal{O}(d)) \cong \oplus^{\left(\begin{array}{c}n+l \\ n\end{array}\right)} \mathcal{O}_{\mathbb{P}}(d-l)$

of locally free sheaves.

Proof. The $P$-modules $\operatorname{Sym}^{1}\left(V^{*}\right)$ corresponds to the free $\mathcal{O P}$-module $\oplus^{\left(\begin{array}{c}n+l \\ n\end{array}\right)} \mathcal{O}_{\mathbb{P}}$. The Corollary now follows from Proposition 3.7.

Let $X=\mathbb{G}(m, m+n)$ and consider the $P$-modules

$L^{d-1} \otimes \operatorname{Sym}^{1}\left(\mathfrak{g} / \mathfrak{p}_{L} \otimes L\right) \subseteq \operatorname{Sym}^{d}\left(\wedge^{m} V\right)$

and

$\mathrm{U}_{1}(\mathfrak{g}) L^{d} \subseteq \operatorname{Sym}^{d}\left(\wedge^{m} V\right)$.

Proposition 3.9. There is an isomorphism

$\mathrm{U}_{1}(\mathfrak{g}) L^{d} \cong L^{d-1} \otimes \operatorname{Sym}^{1}\left(\mathfrak{g} / \mathfrak{p}_{L} \otimes L\right)$

of P-modules.

Proof. Pick an element $x\left(L^{d}\right)=d L^{d-1} x(L) \in U_{1}(\mathfrak{g}) L^{d}$. It follows $d L^{d-1} x(L) \in L^{d-1} \otimes \operatorname{Sym}^{1}\left(\mathfrak{g} / \mathfrak{p}_{L} \otimes L\right)$ hence there is an inclusion

$\mathrm{U}_{1}(\mathfrak{g}) L^{d} \subseteq L^{d-1} \otimes \operatorname{Sym}^{1}\left(\mathfrak{g} / \mathfrak{p}_{L} \otimes L\right)$.

Let $L^{d-1} x(L) \in L^{d-1} \otimes \operatorname{Sym}^{1}\left(\mathfrak{g} / \mathfrak{p}_{L} \otimes L\right)$. It follows

$$
L^{d-1} x(L)=\frac{1}{d} x\left(L^{d}\right) \in \mathrm{U}_{1}(\mathfrak{g}) L^{d}
$$

hence there is an inclusion $L^{d-1} \otimes \operatorname{Sym}^{1}\left(\mathfrak{g} / \mathfrak{p}_{L} \otimes L\right)$ and the Proposition is proved.

Corollary 3.10. There is an isomorphism

$$
\mathcal{P}_{X}^{1}\left(\mathcal{O}_{X}(d)\right)(x)^{*} \cong L^{d-1} \otimes \operatorname{Sym}^{1}\left(\mathfrak{g} / \mathfrak{p}_{L} \otimes L\right)
$$

of P-modules.

Proof. There is by studies of Makestad [1], Theorem 3.10 an isomorphism

$$
\mathcal{P}_{X}^{1}\left(\mathcal{O}_{X}(d)\right)(x)^{*} \cong \mathrm{U}_{1}(\mathfrak{g}) L^{d}
$$

of $P$-modules. The Corollary follows from this fact and Proposition 5.1.

Note: By studies of Maakestad [11], Example 5.12 there is a double complex

$$
\mathcal{O}_{X}(j) \otimes \mathrm{H}^{i}\left(X, \wedge^{j} \mathcal{P}_{X}^{l}\left(\mathcal{O}_{X}(d)\right)^{*}\right)
$$

of sheaves on $\mathcal{P}\left(W^{*}\right)$ where $W=\mathrm{H}^{0}\left(X, \mathcal{O}_{X}(d)\right)$ and $X=\mathbb{G}(m, m+n)$. This double complex might give rise to a resolution of the ideal sheaf of the $l^{\prime}$ th discriminant $D^{l}\left(\mathcal{O}_{X}(d)\right) \subseteq \mathbb{P}\left(W^{*}\right)$ of the line bundle $\mathcal{O}_{X}(d)$. By the literature of Maakestad, Theorem 5.2 it follows knowledge on the $P$-module structure of $\mathcal{P}_{Y}^{l}\left(\mathcal{O}_{Y}(d)\right)$ gives information on the $\operatorname{SL}(V)$-module structure of the higher cohomology groups $\mathrm{H}^{i}\left(X, \wedge^{j} \mathcal{P}_{X}^{l}\left(\mathcal{O}_{X}(d)\right)^{*}\right)$ for all $i \geq 0$. This again gives information on the dimension $h^{i}\left(X, \wedge^{j} \mathcal{P}_{X}^{l}\left(\mathcal{O}_{X}(d)\right)^{*}\right)$. We get a description of the locally free sheaf

$$
\mathcal{O}_{X}(j) \otimes \mathrm{H}^{i}\left(X, \wedge^{j} \mathcal{P}_{X}^{l}\left(\mathcal{O}_{X}(d)\right)^{*}\right) .
$$

for all $i, j$.

Example 3.11. Canonical filtration for the grassmannian $\mathbb{G}(2,4)$.

Consider the example where $m=n=2$ and $X=\mathbb{G}(2,4)$. We get two inclusions

$$
L^{d-2} \otimes \operatorname{Sym}^{2}\left(\mathfrak{g} / \mathfrak{p}_{L} \otimes L\right) \subseteq \operatorname{Sym}^{d}\left(\wedge^{2} V\right)
$$

and

$\mathrm{U}_{2}(\mathfrak{g}) L^{d} \subseteq \operatorname{Sym}^{d}\left(\wedge^{2} V\right)$.

We may choose a basis for $\mathfrak{p} \subseteq \mathfrak{g}$ on the following form:

$\mathfrak{p}=\mathfrak{p}_{L} \oplus L_{x}$

where $L_{x}$ is the line spanned by the following vector $x$ :

$$
x=\left(\begin{array}{cccc}
0 & 0 & 0 & 0 \\
0 & 1 & 0 & 0 \\
0 & 0 & -1 & 0 \\
0 & 0 & 0 & 0 .
\end{array}\right)
$$

Let $\mathfrak{n} \subseteq \mathfrak{g}$ be the sub Lie algebra spanned by the following vectors:

$$
\begin{aligned}
& x_{1}=\left(\begin{array}{llll}
0 & 0 & 0 & 0 \\
0 & 0 & 0 & 0 \\
1 & 0 & 0 & 0 \\
0 & 0 & 0 & 0
\end{array}\right) \\
& x_{2}=\left(\begin{array}{llll}
0 & 0 & 0 & 0 \\
0 & 0 & 0 & 0 \\
0 & 1 & 0 & 0 \\
0 & 0 & 0 & 0
\end{array}\right)
\end{aligned}
$$




$$
x_{3}=\left(\begin{array}{llll}
0 & 0 & 0 & 0 \\
0 & 0 & 0 & 0 \\
0 & 0 & 0 & 0 \\
1 & 0 & 0 & 0
\end{array}\right)
$$

and

$$
x_{4}=\left(\begin{array}{llll}
0 & 0 & 0 & 0 \\
0 & 0 & 0 & 0 \\
0 & 0 & 0 & 0 \\
0 & 1 & 0 & 0
\end{array}\right) .
$$

Let $\tilde{\mathfrak{n}}$ be the vector space spanned by the vectors $x_{1}, x_{2}, x_{4}, x_{4}$ and $x$. It follows $\mathrm{U}_{2}(\mathfrak{g}) L^{d}=\mathrm{U}_{2}(\tilde{\mathfrak{n}}) L^{d} \subseteq \operatorname{Sym}^{d}\left(\wedge^{2} V\right)$. The vector space $V$ has a basus $e_{1}, e_{2}, e_{3}$ and $e_{4}$. The vector space $W$ has basis $e_{1}, e_{2}$. It follows $\wedge^{2} W$ has a basis given by $e_{1} \wedge e_{2}=\mathrm{e}[12]$ and $\wedge^{2} V$ has basis given by $e[12], e[13], e[14], e[23], e[24], e[34]$. By definition $L=e[12]$. We get the following calculation:

$$
\begin{aligned}
& x_{1}(L)=-e[23], x_{2}(L)=e[13], x_{3}(L)=-e[24] \\
& x_{4}(L)=e[14], x(L)=e[12] .
\end{aligned}
$$

A basis for the $P$-module $L^{d-2} \otimes \operatorname{Sym}^{2}\left(\mathfrak{g} / \mathfrak{p}_{L} \otimes L\right)$ are the following vectors:

$$
\begin{aligned}
& L^{d-2} x(L) x(L)=L^{d-2} e[12]^{2} \\
& L^{d-2} x_{2}(L) x(L)=L^{d-2} e[12] e[13] \\
& L^{d-2} x_{4}(L) x(L)=L^{d-2} e[12] e[14] \\
& L^{d-2} x_{1}(L) x(L)=-L^{d-2} e[12] e[23] \\
& L^{d-2} x_{3}(L) x(L)=-L^{d-2} e[12] e[24] \\
& L^{d-2} x_{2}(L) x_{2}(L)=L^{d-2} e[13]^{2} \\
& L^{d-2} x_{2}(L) x_{4}(L)=L^{d-2} e[13] e[14] \\
& L^{d-2} x_{1}(L) x_{2}(L)=-L^{d-2} e[13] e[23] \\
& L^{d-2} x_{2}(L) x_{3}(L)=-L^{d-2} e[13] e[24] \\
& L^{d-2} x_{4}(L) x_{4}(L)=-L^{d-2} e[14]^{2} \\
& L^{d-2} x_{1}(L) x_{4}(L)=-L^{d-2} e[14] e[23] \\
& L^{d-2} x_{3}(L) x_{4}(L)=-L^{d-2} e[14] e[24] \\
& L^{d-2} x_{1}(L) x_{1}(L)=L^{d-2} e[23]^{2} e \\
& L^{d-2} x_{1} \ddot{u}\left[i \ddot{i} \ddot{u}_{3} \ddot{i} i \quad L^{d-2} e \quad e\right. \\
& L^{d-2} x_{3}(L) x_{3}(L)=L^{d-2} e[24]^{2}
\end{aligned}
$$

Let $a=d(d-1)$. A basis for the $P$-module $\mathrm{U}_{2}(\mathfrak{g}) L^{d}=\mathrm{U}_{2}(\tilde{\mathfrak{n}}) L^{d}$ are the following vectors:

$$
\begin{aligned}
& x^{2}\left(L^{d}\right)=L^{d-2} e[12]^{2} \\
& x_{2} x\left(L^{d}\right)=a L^{d-2} e[12] e[13]+d L^{d-1} e[13] \\
& x_{4} x\left(L^{d}\right)=a L^{d-2} e[12] e[14]+d L^{d-1} e[14] \\
& x_{1} x\left(L^{d}\right)=a L^{d-2} e[12] e[23]-d L^{d-1} e[23] \\
& x_{3} x\left(L^{d}\right)=a L^{d-2} e[12] e[24]-d L^{d-1} e[24] \\
& x_{2}^{2}\left(L^{d}\right)=a L^{d-2} e[13]^{2} \\
& x_{2} x_{4}\left(L^{d}\right)=a L^{d-2} e[13] e[14] \\
& x_{1} x_{2}\left(L^{d}\right)=a L^{d-2} e[13] e[23]
\end{aligned}
$$

$$
\begin{aligned}
& x_{2} x_{3}\left(l^{D}\right)=-a L^{d-2} e[13] e[24]-d L^{d-1} e[34] \\
& x_{4}^{2}\left(L^{d}\right)=L^{d-2} e[14]^{2} \\
& x_{1} x_{4}\left(L^{d}\right)=-a L^{d-2} e[14] e[23]+d L^{d-1} e[34] \\
& x_{3} x_{4}\left(L^{d}\right)=-a L^{d-2} e[14] e[24] \\
& x_{1}^{2}\left(L^{d}\right)=a L^{d-2} e[23]^{2} \\
& x_{1} x_{3}\left(L^{d}\right)=a L^{d-2} e[23] e[24] \\
& x_{3}^{2}\left(L^{d}\right)=a L^{d-2} e[24]^{2} .
\end{aligned}
$$

In the case where $W \subseteq V$ have dimensions $m$ and $m+n$ we get embeddings of $P$-modules

$$
\mathrm{U}_{l}(\mathfrak{g}) L^{d} \subseteq \operatorname{Sym}^{d}\left(\wedge^{m} V\right)
$$

and

$$
L^{d-l} \otimes \operatorname{Sym}^{l}\left(\mathfrak{g} / \mathfrak{p}_{L} \otimes L\right) \subseteq \operatorname{Sym}^{d}\left(\wedge^{m} V\right) .
$$

There is no equality

$\mathrm{U}_{l}(\mathfrak{g}) L^{d}=L^{d-l} \otimes \operatorname{Sym}^{l}\left(\mathfrak{g} / \mathfrak{p}_{L} \otimes L\right)$

of $P$-modules as submodules of $\operatorname{Sym}^{d}\left(\wedge^{m} V\right)$ in general as Example 3.11 shows.

Since $\mathrm{U}_{l}(\mathrm{~g}) L^{d}$ and $L^{d-l} \otimes \operatorname{Sym}^{l}\left(\mathfrak{g} / \mathfrak{p}_{L} \otimes L\right)$ by Theorem 3.5 and Proposition 3.3 are isomorphic when $m=1$ and $1 \leq l \leq d$, have the same dimension over $K$ and both have natural filtrations of $P$-modules we may conjecture they are isomorphic as $P$-modules for all $m, n \geq 1$. Note: There is a canonical line $L^{d} \in \mathrm{U}_{l}(\mathfrak{g}) L^{d}$ for all $l$. There is similarly a canonical line

$$
L^{d} \cong L^{d-l} \otimes \operatorname{Sym}^{l}\left(\mathfrak{p} / \mathfrak{p}_{L} \otimes L\right) \in L^{d-l} \otimes \operatorname{Sym}^{l}\left(\mathfrak{g} / \mathfrak{p}_{L} \otimes L\right) .
$$

Hence the two $P$-modules $\mathrm{U}_{l}(\mathrm{~g}) L^{d}$ and $L^{d-l} \otimes \operatorname{Sym}^{l}\left(\mathfrak{g} / \mathfrak{p}_{L} \otimes L\right)$ look similar.

In general the $\operatorname{SL}(V)$-module $\operatorname{Sym}^{d}\left(\wedge^{m} V\right)$ decompose

$\operatorname{Sym}^{d}\left(\wedge^{m} V\right) \cong \oplus_{i} V_{\lambda_{i}}^{a_{i}}$

where $V_{\lambda_{i}}$ are irreducible SL(V)-modules and $a_{i} \geq 1$ are integers (Proposition 5.4 for the situation of $\mathbb{G}(2,4)$. One may ask if there is a non-trivial automorphism

$\phi \in \operatorname{Aut}_{\mathrm{SL}(V)}\left(\operatorname{Sym}^{d}\left(\wedge^{m} V\right)\right)$

with the property that the morphism

$\phi: \operatorname{Sym}^{d}\left(\wedge^{m} V\right) \rightarrow \operatorname{Sym}^{d}\left(\wedge^{m} V\right)$

induce an isomorphism

$\tilde{\phi}: L^{d-l} \otimes \operatorname{Sym}^{l}\left(\mathfrak{g} / \mathfrak{p}_{L} \otimes L\right) \rightarrow \mathrm{U}_{l}(\mathfrak{g}) L^{d}$

of $P$-modules. In general the $\operatorname{SL}(V)$-module $\operatorname{Sym}^{d}\left(\Lambda^{m} V\right)$ has lots of automorphisms. When $m=2$ and $\operatorname{dim}_{k}(V)=4$ it follows by Corollary 5.4 there is for every $d \geq 1$ an equality

$$
\operatorname{Aut}_{\mathrm{SL}(V)}\left(\operatorname{Sym}^{d}\left(\wedge^{2} V\right)\right)=\prod_{i=0}^{l} K^{*}
$$

where $l=k$ if $d=2 k$ or $d=2 k+1$. For $m=n=2$ the $\operatorname{SL}(V)$-module $\operatorname{Sym}^{d}\left(\wedge^{m} V\right)$ is by Proposition 5.4 multiplicity free. The module $\operatorname{Sym}^{d}\left(\wedge^{m} K^{m+n}\right)$ is not multiplicity free in general when $m, n>2$.

\section{Jet Bundles and Incidence Complexes on the Projective Line}

In this section we construct a resolution by locally free sheaves 
of the ideal sheaf of the $l^{l}$ th incidence scheme $I^{l}\left(\mathcal{O}_{\mathbb{p}}(d)\right) \subseteq \mathbb{P}\left(W^{*}\right) \times \mathbb{P}$. Here $\mathcal{O P}(d)$ is an invertible sheaf on the projective line $\mathcal{P}=\mathcal{P}^{1}$ and $W=\mathrm{H}^{0}\left(\mathbb{P}, \mathcal{O}_{p}(d)\right)$. There is on $Y=\mathcal{P}\left(W^{*}\right) \times \mathcal{P}^{1}$ a morphism $\varphi(\mathcal{O}(d))$ of locally free sheaves

$$
\phi(\mathcal{O}(d)): \mathcal{O}_{\mathbb{P}\left(W^{*}\right)}(-1)_{Y} \rightarrow \mathcal{P}^{l}(\mathcal{O}(d))_{Y}
$$

Its zero scheme $Z(\phi(\mathcal{O}(d)))=I^{l}(\mathcal{O}(d)) \subseteq Y$ is the $l$ th incidence scheme of $\mathcal{O}(d)$. The Koszul complex of the morphism $\varphi(\mathcal{O}(d))$

$$
\begin{aligned}
& 0 \rightarrow \wedge^{l} \mathcal{O}(-1)_{Y} \otimes \mathcal{P}^{l}(\mathcal{O}(d))_{Y}^{*} \rightarrow \cdots \rightarrow \wedge^{2} \mathcal{O}(-1)_{Y} \otimes \mathcal{P}^{l}(\mathcal{O}(d))_{Y}^{*} \rightarrow \\
& \mathcal{O}(-1)_{Y} \otimes \mathcal{P}^{l}(\mathcal{O}(d))_{Y}^{*} \rightarrow \mathcal{O}_{Y} \rightarrow \mathcal{O}_{I^{l}(\mathcal{O}(d))} \rightarrow 0
\end{aligned}
$$

- called the incidence complex of $\mathcal{O}(d)$ - is a resolution of the ideal sheaf of $I^{l}(\mathcal{O}(d))$. This follows from the fact that the ideal sheaf of $I$ ${ }^{l}(\mathcal{O}(d))$ is locally generated by a regular sequence. We also calculate the higher direct images of the terms

$$
\mathcal{O}(-j)_{Y} \otimes \wedge^{j} \mathcal{P}^{l}(\mathcal{O}(d))_{Y}^{*}
$$

appearing in the incidence complex.

The aim of the construction is to use it to construct a resolution of the ideal sheaf of the discriminant $D^{l}(\mathcal{O}(d))$ where $\mathcal{O}(d)$ is a line bundle on projective space or a grassmannian.

Example 4.1. The Koszul complex of a map of locally free modules.

Let $A$ be an arbitrary commutative ring with unit and let $\varphi: E \rightarrow F$ be a map $A$-modules.

Define the following map:

$$
d^{0}: E \otimes_{A} F^{*} \rightarrow A
$$

by

$$
d^{0}(x \otimes f)=f(\phi(x)) .
$$

Let $I A$ be the image of $d^{1}$. We let $I_{\varphi}$ be the ideal of $\varphi$. Define the following map

$$
d^{p}: \wedge^{p} E \otimes F^{*} \rightarrow \wedge^{p-1} E \otimes F^{*}
$$

by

$$
d^{p}\left(x_{1} \otimes f_{1} \wedge \cdots \wedge x_{p} \otimes f_{p}\right)=\sum_{r=1}^{p}(-1)^{r-1} f_{r}\left(\phi\left(x_{r}\right)\right) x_{1} \otimes f_{1} \wedge \cdots \wedge \widehat{x_{r} \otimes f_{r}} \wedge \cdots \wedge x_{p} \otimes f_{p} .
$$

Lemma 4.2. The following holds for all $p \geq 1: d^{p}$ o $d^{p-1}=0$.

Proof. We get

$$
\begin{aligned}
& d^{p-1} d^{p}\left(x_{1} \otimes f_{1} \wedge \cdots \wedge x_{p} \otimes f_{p}\right)= \\
& \sum_{r=1}^{p}(-1)^{r-1} f_{r}\left(\phi\left(x_{r}\right)\right) \\
& \sum_{l \neq r}(-1)^{l-1} f_{l}\left(\phi\left(x_{l}\right)\right) x_{1} \otimes f_{1} \wedge \cdots \wedge \overline{x_{l} \otimes f_{l}} \wedge \cdots \wedge \overline{x_{r} \otimes f_{r}} \wedge \cdots \wedge x_{p} \otimes f_{p}=0
\end{aligned}
$$

and the claim of the Lemma follows.

Assume $E, F$ are locally free of finite rank and let $r=r k\left(E \otimes F^{*}\right)$. We get a complex of locally free $A$-modules

$$
0 \rightarrow \wedge^{r} E \otimes F^{*} \rightarrow \cdots \rightarrow \wedge^{2} E \otimes F^{*} \rightarrow E \otimes F^{*} \rightarrow A \rightarrow A / I_{\phi} \rightarrow 0
$$

called the Koszul complex of the map $\varphi$

Example 4.3. The Koszul complex of a regular sequence.

Let $\underline{x}=\left\{x_{1}, . ., x_{n}\right\}$ be a regular sequence of elements in $A$ and let $E=$ $A e$ be the free $A$-module on the element $e$. Let $F=A\left\{e_{1}, . ., e_{n}\right\}$ be a free rank $n$ module on $e_{1}, . ., e_{n}$. Let $y_{i}=e_{i}^{*}$. Define

$$
\varphi: E \rightarrow F
$$

by

$$
\phi(e)=x_{1} e_{1}+\cdots+x_{n} e_{n} .
$$

Let $e \otimes y_{i}=z_{i}$. It follows

$d^{p}: \wedge^{p} E \otimes F^{*} \rightarrow \wedge^{p-1} E \otimes F^{*}$

looks as follows:

$$
\begin{aligned}
& d^{p}\left(z_{i_{1}} \wedge \cdots \wedge z_{i_{p}}\right)= \\
& \sum_{r=1}^{p}(-1)^{p-1} y_{i_{r}}(\phi(e)) z_{i_{1}} \wedge \cdots \wedge \widetilde{z_{i_{r}}} \wedge \cdots \wedge z_{i_{p}}= \\
& \sum_{r=1}^{p}(-1)^{r-1} x_{i_{r}} z_{i_{1}} \wedge \cdots \wedge \widetilde{z_{i_{r}}} \wedge \cdots \wedge z_{i_{p}} .
\end{aligned}
$$

Hence the complex $\wedge^{\bullet} E \otimes F^{*}$ equals the Koszul complex $K_{.}(\underline{x})$ of the regular sequence $\underline{x}$. It is an exact complex since $\underline{x}$ is a regular sequence.

Example 4.4. The Koszul complex of a morphism of locally free sheaves.

The construction of the differential in the Koszul complex of a map of modules is intrinsic, hence we may generalize to morphisms of locally free sheaves. Let $Y$ be an arbitrary scheme and let $\varphi: \mathcal{E} \rightarrow \mathcal{F}$ be a map of locally free $\mathcal{O}_{Y}$-modules. Let

$d^{0}: \mathcal{E} \otimes \mathcal{F}^{*} \rightarrow \mathcal{O}_{Y}$

be defined locally by

$d^{0}(s \otimes v)=v(\phi(s))$.

Let $\mathcal{I}_{\phi}=\operatorname{Im}\left(d^{0}\right) \subseteq \mathcal{O}_{Y}$ be the ideal sheaf defined by $d^{1}$. Since $\mathcal{I}_{\varphi}$ is quasi coherent sheaf of ideals it follows the ideal sheaf $\mathcal{I}_{\varphi}$ corresponds to a subscheme $Z(\varphi) \subseteq Y$ - the zero scheme of $\varphi$. Let $U \subseteq Y$ be an open subset and define the following map:

$$
d^{p}: \wedge^{p}\left(\mathcal{E} \otimes \mathcal{F}^{*}\right)(U) \rightarrow \wedge^{p-1}\left(\mathcal{E} \otimes \mathcal{F}^{*}\right)(U)
$$

by

$$
d^{p}\left(s_{1} \otimes v_{1} \wedge \cdots \wedge s_{p} \otimes v_{p}\right)=\sum_{r=1}^{p}(-1)^{r-1} v_{r}\left(\phi\left(s_{r}\right)\right) s_{1} \otimes v_{1} \wedge \cdots \wedge \widehat{s_{r} \otimes v_{r}} \wedge \cdots \wedge s_{p} \otimes v_{p} .
$$

This gives a well defined map of locally free sheaves since we have not chosen a basis for the module $\wedge^{p}\left(\mathcal{E} \otimes \mathcal{F}^{*}\right)(U)$ to give a definition. By Lemma 4.2 it follows $d^{p}$ o $d^{p+1}=0$ for all $p \geq 1$ hence we get a complex of locally free sheaves. The sequence of maps of locally free sheaves

$0 \rightarrow \wedge^{r} \mathcal{E} \otimes \mathcal{F}^{*} \rightarrow \cdots \rightarrow \wedge^{2} \mathcal{E} \otimes \mathcal{F}^{*} \rightarrow \mathcal{E} \otimes \mathcal{F}^{*} \rightarrow \mathcal{O}_{Y} \rightarrow \mathcal{O}_{Z(\phi)} \rightarrow 0$

is called the Koszul complex of $\phi$. Here $r=r k(\mathcal{E} \otimes \mathcal{F})$.

Example 4.5. Koszul complexes and local complete intersections.

Assume $\phi: \mathcal{L} \rightarrow \mathcal{F}$ is a map of locally free $\mathcal{O}_{Y}$-modules where $\mathcal{L}$ is a line bundle. Let $Z(\phi) \subseteq Y$ be the subscheme defined by $\phi$ - the zero scheme of $\phi$. Let $r=r k(\mathcal{F})$. Choose an open affine cover $\mathrm{U}_{i}$ of $Y$ where $\mathcal{F}$ and $\mathcal{L}$ trivialize, i.e

$$
\mathcal{F}\left(U_{i}\right)=\mathcal{O}\left(U_{i}\right)\left\{f_{i 1}, . ., f_{i r}\right\}
$$

and

$\mathcal{L}\left(U_{i}\right)=\mathcal{O}\left(U_{i}\right) e_{i}$

Let $\mathcal{O}\left(U_{i}\right)=A_{i}, L_{i}=\mathcal{L}\left(U_{i}\right)$ and $F_{i}=\mathcal{F}\left(U_{i}\right)$. Assume the image 


$$
\phi\left(U_{i}\right): L_{i} \rightarrow F_{i}
$$

has

$$
\phi\left(U_{i}\right)\left(e_{i}\right)=x_{i 1} f_{i 1}+\cdots+x_{i r} f_{i r}
$$

where $\left\{x_{i 1}, . ., x_{i r}\right\} \subseteq A_{i}$ is a regular sequence. Let $I_{i}=\underline{x_{i}}=\left\{x_{i 1}, . ., x_{i r}\right\}$. It follows from Example 4.3 the Koszul complex

$$
\begin{aligned}
& 0 \rightarrow \wedge^{\prime}\left(L_{i} \otimes F_{i}^{*}\right) \rightarrow \cdots \rightarrow \wedge^{2}\left(L_{i} \otimes F_{i}^{*}\right) \rightarrow L_{i} \otimes F_{i}^{*} \\
& \rightarrow A_{i} \rightarrow A_{i} / I_{i} \rightarrow 0
\end{aligned}
$$

is a resolution of the ideal $I_{i}$ since $I_{i}$ is generated by a regular sequence. The complex $\wedge^{*} L_{i} \otimes F_{i}^{*}$ is isomorphic to the Koszul complex $K .\left(x_{i}\right)$ on the regular sequence $\underline{x_{i}}$. It follows the global complex

$$
0 \rightarrow \mathcal{L}^{\otimes r} \wedge^{r} \mathcal{F}^{*} \rightarrow \cdots \rightarrow \mathcal{L}^{\otimes 2} \wedge^{2} \mathcal{F}^{*} \rightarrow \mathcal{L} \otimes \mathcal{F}^{*} \rightarrow \mathcal{O}_{Y} \rightarrow \mathcal{O}_{Z(\phi)} \rightarrow 0
$$

is a resolution of the ideal sheaf $\mathcal{I}_{Z(\varphi)}$ of $Z(\varphi) \subseteq Y$ since it is locally isomorphic to the Koszul complex $K .\left(x_{i}\right)$ for all $i$.

Since the ideal $I_{i}$ is generated by a regular sequence of length $r$ it follows $\operatorname{dim}\left(A_{i} / I_{i}\right) \stackrel{\operatorname{dim}}{=}\left(A_{i}\right)-r$. If $Y$ is irreducible of dimension $d$ it follows $Z(\varphi) \subseteq Y$ is a local complete intersection of dimension $d-r$.

Example 4.6. The incidence complex of $\mathcal{O}(d)$ on the projective line.

Let $\mathbb{P}=\mathbb{P}_{K}^{1}$ where $K$ is a field of characteristic zero and let $\mathcal{O}(d) \in$ $\operatorname{Pic}(\mathcal{P})=\mathrm{Z}$ be a line bundle where $d \in \mathrm{Z}$. Let

$$
W=\mathrm{H}^{0}(\mathbb{P}, \mathcal{O}(d))=K\left\{e_{0}, . ., e_{d}\right\}
$$

where $e_{i}=x_{0}^{d-i} x_{1}^{i}$. Let $y_{i}=e_{i}^{*}$. Let $Y=\mathcal{P}\left(W^{*}\right) \times \mathcal{P}$ and consider the following diagram

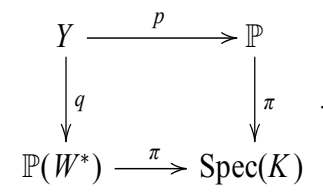

There is a sequence of locally free $\mathcal{O}_{Y}$-modules

$$
\mathcal{O}_{\mathbb{P}\left(W^{*}\right)}(-1)_{Y} \rightarrow \mathrm{H}^{0}(\mathbb{P} . \mathcal{O}(d)) \otimes \mathcal{O}_{Y} \rightarrow^{T_{Y}^{l}} \mathcal{P}^{l}(\mathcal{O}(d))_{Y}
$$

and let $\varphi(\mathcal{O}(d))$ be the composed map

$$
\phi(\mathcal{O}(d)): \mathcal{O}_{\mathbb{P}\left(W^{*}\right)}(-1)_{Y} \rightarrow \mathcal{P}^{l}(\mathcal{O}(d))_{Y} .
$$

It follows by studies of Maakestad [11], the zero scheme $Z(\varphi$ $(\mathcal{O}(d))$ ) equals the incidence scheme $I^{l}(\mathcal{O}(d))$ of the line bundle $\mathcal{O}(d)$. By definition $\mathbb{P}\left(W^{*}\right)=\operatorname{Proj}\left(K\left[y_{0}, . ., y_{d}\right]\right)$ where $y_{i}=e_{i}^{*}$. It has an open cover on the following form: $D\left(y_{i}\right)=\operatorname{Spec}\left(K\left[u_{0}, . ., u_{d}\right]\right)$ where we let $u_{j}=\frac{y_{j}}{y_{i}}$. Let $y_{j} / y_{j}=1$. Let

$$
F(t)=u_{0}+u_{1} t+\cdots+u_{d} t^{d} \in K\left[u_{0}, \ldots, u_{d}, t\right] .
$$

Restrict the map 4.6.1 to the open set $U_{i 0}=D\left(y_{i}\right) \times D\left(x_{0}\right) \subseteq Y$. We get the following two maps of modules:

$$
\begin{aligned}
& \alpha:\left.\mathcal{O}_{\mathbb{P}\left(W^{*}\right)}(-1)\right|_{U_{i 0}} \rightarrow \mathcal{O}_{U_{i 0}} \otimes \mathrm{H}^{0}(\mathbb{P}, \mathcal{O}(d)) \\
& \alpha: K\left[y_{i}, t\right] \frac{1}{y_{i}} \rightarrow K\left[u_{i}, t\right] \otimes K\left\{e_{0}, . ., e_{d}\right\}
\end{aligned}
$$

defined by

$$
\alpha\left(1 / y_{i}\right)=\sum_{k=0}^{d} u_{k} \otimes e_{k}=\sum_{k=0}^{d} u_{k} \otimes x_{0}^{d-k} x_{1}^{k}=\sum_{k=0}^{d} u_{k} \otimes t^{k} x_{0}^{d} .
$$

We get the map
$T_{U_{i 0}}^{l}:\left.\mathcal{O}_{U_{i 0}} \otimes \mathrm{H}^{0}(\mathbb{P}, \mathcal{O}(d)) \rightarrow \mathcal{P}^{l}(\mathcal{O}(d))\right|_{U_{i 0}}$

defined by

$T^{l}\left(1 \otimes x_{0}^{d-i} x_{1}^{i}\right)=T^{l}\left(1 \otimes t^{i} x_{0}^{d}\right)=(t+d t)^{i} \otimes x_{0}^{d}$.

The composed map

$\phi(\mathcal{O}(d))_{U_{i 0}}: K\left[u_{i}, t\right] \frac{1}{y_{i}} \rightarrow K\left[u_{i}, t\right]\left\{d t^{j} \otimes x_{0}^{d}\right\}$

is the map

$\phi(\mathcal{O}(d))\left(\frac{1}{y_{i}}\right)=\sum_{k=0}^{d} u_{k}(t+d t)^{k} \otimes x_{0}^{d}=$

$\sum_{k=0}^{l} \frac{F^{(k)}(t)}{k !} d t^{k} \otimes x_{0}^{d} \in K\left[u_{i}, t\right]\left\{1 \otimes x_{0}^{d}, . ., d t^{l} \otimes x_{0}^{d}\right\}$.

Let $U_{i 1}=D\left(y_{i}\right) \times D\left(x_{1}\right) \subseteq Y$ and let $\frac{x_{0}}{x_{1}}=s$. Let

$G(s)=u_{d}+u_{d-1} s+u_{d-2} s^{2}+\cdots+u_{0} s^{d} \in K\left[u_{0}, . ., u_{d}, s\right]$.

Restrict the map 4.6.1 to the open set $U_{i 1}$

We get the following two maps of modules:

$\alpha:\left.\mathcal{O}_{\mathbb{P}\left(W^{*}\right)}(-1)\right|_{U_{i 1}} \rightarrow \mathcal{O}_{U_{i 1}} \otimes \mathrm{H}^{0}(\mathbb{P}, \mathcal{O}(d))$

$\alpha: K\left[y_{i}, s\right] \frac{1}{y_{i}} \rightarrow K\left[u_{i}, s\right] \otimes K\left\{e_{0}, . ., e_{d}\right\}$

defined by

$\alpha\left(1 / y_{i}\right)=\sum_{k=0}^{d} u_{k} \otimes e_{k}=\sum_{k=0}^{d} u_{k} \otimes x_{0}^{d-k} x_{1}^{k}=\sum_{k=0}^{d} u_{k} \otimes s^{d-k} x_{1}^{d}$.

We get the map

$T_{U_{i 1}}^{l}:\left.\mathcal{O}_{U_{i 1}} \otimes \mathrm{H}^{0}(\mathbb{P}, \mathcal{O}(d)) \rightarrow \mathcal{P}^{l}(\mathcal{O}(d))\right|_{U_{i 1}}$

defined by

$T^{l}\left(1 \otimes x_{0}^{d-i} x_{1}^{i}\right)=T^{l}\left(1 \otimes s^{d-i} x_{1}^{d}\right)=(s+d s)^{d-i} \otimes x_{1}^{d}$.

The composed map

$\phi(\mathcal{O}(d))_{U_{i 1}}: K\left[u_{i}, s\right] \frac{1}{y_{i}} \rightarrow K\left[u_{i}, s\right]\left\{d s^{j} \otimes x_{1}^{d}\right\}$

is the map

$$
\begin{aligned}
& \phi(\mathcal{O}(d))\left(\frac{1}{y_{i}}\right)=\sum_{k=0}^{d} u_{d-k}(s+d s)^{k} \otimes x_{1}^{d}= \\
& \sum_{k=0}^{l} \frac{G^{(k)}(s)}{k !} d s^{k} \otimes x_{1}^{d} \in K\left[u_{i}, s\right]\left\{1 \otimes x_{1}^{d}, . ., d s^{l} \otimes x_{1}^{d}\right\} .
\end{aligned}
$$

It follows the ideal sheaf $\mathcal{I}_{I^{l}(\mathcal{O}(d))}$ of $I^{l}(\mathcal{O}(d))$ is generated by

$$
\left\{\frac{F^{(l)}(t)}{l !}, \frac{F^{(l-1)}(t)}{(l-1) !}, . ., F(t)\right\}
$$

on $U_{i 0}$ and by

$\left\{\frac{G^{(l)}(s)}{l !}, \frac{G^{(l-1)}(s)}{(l-1) !}, . ., G(s)\right\}$

on $U_{i 1}$. Let $z_{i}=\frac{F^{(i)}(t)}{(i) !}$ and $w_{i}=\frac{G^{(i)}(s)}{(i) !}$ for $i=0, . ., l$.

Lemma 4.7. Assume $B$ is a commutative ring of characteristic zero and let

$f(t)=a_{0}+a_{1} t+\cdots+a_{d} t^{d} \in B[t]$

be an arbitrary degree $d$ polynomial with $a_{d} \neq 0$. Let $f^{(i)}(t)$ denote the formal derivative with respect to $t$. It follows 


$$
\frac{f^{(k)}(t)}{l !}=\sum_{i=k}^{d}\left(\begin{array}{l}
i \\
k
\end{array}\right) a_{i} t^{i-k}
$$

Proof. The proof is by induction. It is clearly true for $l=1$. Assume it is true for $l>1$. Consider $k=l+1$. We get

$$
\begin{aligned}
& \frac{f^{(l+1)}(t)}{(l+1) !}=\frac{1}{l+1} \frac{\partial}{\partial} \frac{f^{(l)(t)}}{l !}= \\
& \left.\frac{1}{l+1}\left(\begin{array}{c}
l+1 \\
l
\end{array}\right) a_{l+1}+\left(\begin{array}{c}
l+2 \\
l
\end{array}\right) 2 a_{l+2} t+\cdots+\left(\begin{array}{l}
d \\
l
\end{array}\right)(d-l) a_{d} t^{d-(l+1)}\right)= \\
& \left(\begin{array}{c}
l+1 \\
l+1
\end{array}\right) a_{l+1}+\left(\begin{array}{c}
l+2 \\
l+1
\end{array}\right) a_{l+2} t+\cdots+\left(\begin{array}{c}
d \\
l+1
\end{array}\right) a_{d} t^{d-(l+1)}= \\
& \sum_{i=l+1}^{d}\left(\begin{array}{c}
i \\
l+1
\end{array}\right) a_{i} t^{i-(l+1)}
\end{aligned}
$$

and the claim of the Lemma follows.

Lemma 4.8. The sequence $\left\{z_{p}, . ., z_{0}\right\}$ is a regular sequence in $K\left[u_{i}, t\right]$. The sequence $\left\{w_{l}, . ., w_{0}\right\}$ is a regular sequence in $K\left[u_{i}, s\right]$.

Proof. Let $z_{i}=\frac{F^{(i)}(t)}{i !}$ and $w_{j}=\frac{G^{(j)}}{j !}$. Assume $l<i$ and consider the sequence $z_{l}, z_{l-1}, . ., z_{0} \subseteq A[t]=K\left[u_{0}, . ., u_{d}\right][t]$. Since $A[t]$ is a domain it follows $z_{l}$ is a non zero divisor in $A[t]$. We see from Lemma 4.7

$$
A[t] / w_{l} \cong K\left[u_{0}, . ., u_{l-1}, u_{l+1}, . ., u_{d}, t\right]
$$

which is a domain, hence $w_{l-1}$ is a non zero divisor in $A[t] / w_{l}$. By induction it follows $z_{p}, . ., z_{0}$ is a regular sequence in $A[t]$. Assume $i \leq l$. It follows the sequence $z_{l}, . ., z_{i+1}$ is a regular sequence in $A[t]$. We see from Lemma $4.7 z_{l}$ is non zero in

$$
A[t] /\left(z_{l}, . ., z_{i+1}\right)=K\left[u_{0}, . ., u_{i}, u_{l+1}, . ., u_{d}, t\right]
$$

and $K\left[u_{0}, . ., u_{i}, u_{l+1}, . ., u_{d}, t\right]$ is a domain. It follows $z_{i}$ is a non zero divisor in $A[t] /\left(z_{p}, . ., z_{i+1}\right)$. It follows $z_{p} . ., z_{0}$ is a regular sequence in $A[t]$ and the claim follows. A similar argument proves $w_{p}, . ., w_{0}$ is a regular sequence in $A[s]$ and the Lemma is proved.

One may prove using similar methods for any permutation $\sigma \in S_{l+1}$ the sequences

$$
z_{(l)}, \ldots, z_{\sigma(0)}
$$

and

$$
w_{(l)}, \ldots, w_{\sigma(0)}
$$

are regular sequences.

It follows the ideal sheaf $\mathcal{I}_{I^{l}(\mathcal{O}(d))}$ is locally generated by a regular sequence.

The morphism

$\phi(\mathcal{O}(d)): \mathcal{O}_{\mathbb{P}\left(W^{*}\right)}(-1)_{Y} \rightarrow \mathcal{P}^{l}(\mathcal{O}(d))_{Y}$

gives by Example 4.3 rise to a Koszul complex

$\wedge^{\bullet} \mathcal{O}_{\mathbb{P}\left(W^{*}\right)}(-1) \otimes \mathcal{P}^{l}(\mathcal{O}(d))_{Y}^{*}$

of locally free sheaves of $Y=\mathcal{P}\left(W^{*}\right) \times \mathcal{P}^{1}$.

Definition 4.9. Let the complex

$$
\begin{aligned}
& 0 \rightarrow \wedge^{l} \mathcal{O}(-1)_{Y} \otimes \mathcal{P}^{l}(\mathcal{O}(d))_{Y}^{*} \rightarrow \cdots \rightarrow \wedge^{2} \mathcal{O}(-1)_{Y} \otimes \mathcal{P}^{l}(\mathcal{O}(d))_{Y}^{*} \rightarrow \\
& \mathcal{O}(-1)_{Y} \otimes \mathcal{P}^{l}(\mathcal{O}(d))_{Y}^{*} \rightarrow \mathcal{O}_{Y} \rightarrow \mathcal{O}_{I^{l}(\mathcal{O}(d))} \rightarrow 0
\end{aligned}
$$

be the incidence complex of $\mathcal{O}(d)$.

Since the ideal sheaf of $I^{l}(\mathcal{O}(d))$ by the discussion above is locally generated by a regular sequence it follows from Example 4.3 the complex 4.9.1 is a resolution.

In framework of Maakestad [5], Theorem 5.10 one calculates the higer direct images

$$
\mathrm{R}^{i} q_{*}\left(\wedge^{j} \mathcal{O}(-1)_{Y} \otimes \mathcal{P}^{l}(\mathcal{O}(d))_{Y}^{*}\right)
$$

for all $i, j$. We get the following calculations:

Let $V=K\left\{e_{0}, e_{1}\right\}$ and $\mathcal{P}=\mathcal{P}\left(V^{*}\right)$. Let $W=\mathrm{H}^{0}(\mathcal{P}, \mathcal{O}(d))=\operatorname{Sym}^{d}\left(V^{*}\right)$ and consider the diagram

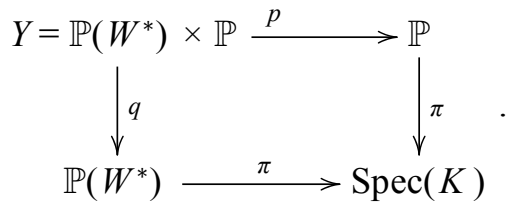

By the results of this paper it follows there is an isomorphism

$$
\mathcal{P}_{\mathbb{P}}^{l}(\mathcal{O}(d)) \cong \mathcal{O}_{\mathbb{P}}(d-l) \otimes \pi^{*} \operatorname{Sym}^{l}\left(V^{*}\right)
$$

a sheaves with an $\operatorname{SL}(V)$-linearization. We get

$\wedge^{j} \mathcal{P}_{\mathbb{P}}^{l}\left(\mathcal{O}_{\mathbb{P}}(d)\right) \cong \mathcal{O}_{\mathbb{P}}(j(d-l)) \otimes \pi^{*} \wedge^{j} \operatorname{Sym}^{l}\left(V^{*}\right)$.

By the equivariant projection formula for higher direct images we get $\mathrm{R}^{i} q_{*}\left(\wedge^{j} \mathcal{O}(-1)_{Y} \otimes \mathcal{P}^{l}(\mathcal{O}(d))_{Y}^{*}\right) \cong \mathcal{O}_{\mathbb{P}\left(W^{*}\right)}(-j) \otimes \mathrm{H}^{i}\left(\mathbb{P}, \wedge^{j} \mathcal{P}_{\mathbb{P}}^{l}\left(\mathcal{O}_{\mathbb{P}}(d)\right)^{*}\right)$.

Let

$\pi: \mathcal{P} \rightarrow \operatorname{Spec}(K)$.

It follows

$\wedge^{j} \mathcal{P}_{\mathbb{P}}^{l}\left(\mathcal{O}_{\mathbb{P}}(d)\right)^{*} \cong \mathcal{O}_{\mathbb{P}}(j(l-d)) \otimes \pi^{*} \wedge^{j} \operatorname{Sym}^{l}\left(V^{*}\right)$.

We get

$\mathrm{H}^{i}\left(\mathbb{P}, \wedge^{j} \mathcal{P}^{l}(\mathcal{O}(d))^{*}\right) \cong \mathrm{R}^{i} \pi_{*}\left(\pi^{*}\left(\wedge^{j} \operatorname{Sym}^{l}(V)\right) \otimes \mathcal{O}_{\mathbb{P}}(j(l-d))\right) \cong$

$\wedge^{j}\left(\operatorname{Sym}^{l}(V)\right) \otimes \mathrm{H}^{i}\left(\mathbb{P}, \mathcal{O}_{\mathbb{P}}(j(l-d))\right)$.

We get the following Theorem:

Theorem 4.10. The following holds:

$\mathrm{R}^{i} p_{*}\left(\mathcal{O}(-j) \otimes \wedge^{j} \mathcal{P}^{l}(\mathcal{O}(d))^{*}\right)=0$ if $i=0$ or $i=1$ and $j(d-l)<2$.

$\mathrm{R}^{1} p_{*}\left(\mathcal{O}(-j) \otimes \wedge^{j} \mathcal{P}^{l}(\mathcal{O}(d))^{*}\right)=\mathcal{O}(-j) \otimes \operatorname{Sym}^{j(d-l)-2}(V) \otimes \wedge^{j} \operatorname{Sym}^{l}(V)$

if $j(d-l) \geq 2$.

Proof. The proof follows from the calculation of the equivariant cohomology of line bundles on projective space [13].

Hence we have complete control on the sheaf

$\mathrm{R}^{i} q_{*}\left(\wedge^{j} \mathcal{O}(-1)_{Y} \otimes \mathcal{P}^{l}(\mathcal{O}(d))_{Y}^{*}\right)$

on the projective line and projective space for all $i, j$. Using the techniques introduced in this paper one may describe resolutions of incidence schemes $I^{l}(\mathcal{O}(d))$ on more general grassmannians and flag varieties. The hope is we may be able to construct resolutions of the ideal sheaf of $D^{l}(\mathcal{O}(d))$ using indicence resolutions in a more general situation.

Note: In literature of Lascoux [12] resolutions of ideal sheaves of determinantal schemes are studied and much is known on such 
resolutions. In studies of Maakestad [11] it is proved $D^{l}(\mathcal{O}(d)$ is a determinantal scheme for any $d \geq 2$ on the projective line $\mathcal{P P}$. Assume $\mathcal{L} \in \operatorname{Pic}^{G}(G / P)$ is a $G$-linearized linebundle, $G$ a semi simple linear algebraic group and $P$ a parabolic subgroup. If one can prove $D$ ${ }^{l}(\mathcal{L})$ is a determinantal scheme we get two approaches to the study of resolutions of ideal sheaves of discriminants: One using jet bundles and incidence schemes, another one using determinantal schemes.

\section{Appendix A: Automorphisms of Representations}

Let $W \subseteq V$ be vectorspaces of dimension two and four over the field $K$. Consider the subgroup $P \subseteq G=\operatorname{SL}(V)$ where $P$ is the parabolic subgroup of elements fixing $W$. It follows $\pi: G \rightarrow G / P=\mathbb{G}(2,4)$ is a principal $P$-bundle. Let $\mathfrak{g}=\operatorname{Lie}(G)$ and $\mathfrak{p}=\operatorname{Lie}(P)$ be the Lie algebras of $G$ and $P$. In this section we study the decomposition into irreducibles and automorphisms of some $G$-modules. We also study some $P_{\text {semi }}$-modules where $P_{\text {semi }}$ is the semi-simplification of $P$. It follows $P_{\text {semi }}$ equals $\operatorname{SL}(2)$ $\times \mathrm{SL}(2)$. Since $\mathfrak{p} \subseteq \mathfrak{g}$ is a $P$-sub module it follows the quotient $\mathfrak{g} / \mathfrak{p}$ is a $P$-module hence a $P_{\text {semi }}$ module. We may apply the theory of highest weights since $P_{\text {semi }}=\mathrm{SL}(2) \times \mathrm{SL}(2)$ is a semi simple algebraic group.

Proposition 5.1. The following hold: There is an isomorphism of $\mathrm{SL}(2) \times \mathrm{SL}(2)$-modules

$$
\operatorname{Sym}^{k}(\mathfrak{g} / \mathfrak{p})=\oplus_{i=0}^{n} \operatorname{Sym}^{2 i+m}\left(W^{*}\right) \otimes \operatorname{Sym}^{2 i+m}(V / W) .
$$
for all $k \geq 1$. Here $(n, m)=(-, 0)$ if $k=2 n$ and $(n, m)=\left(\frac{k-1}{2}, 1\right)$ if $k=2 n$

Proof. Recall the canonical isomorphism from Lemma 2.4

$\mathfrak{g} / \mathfrak{p} \cong \operatorname{Hom}(W, V / W) \cong W^{*} \otimes V / W$

of $P$-modules. It follows

$\operatorname{Sym}^{k}(\mathfrak{g} / \mathfrak{p}) \cong \operatorname{Sym}^{k}\left(W^{*} \otimes V / W\right)$

and its decomposition into irreducible $\mathrm{SL}(2) \times \mathrm{SL}(2)$-modules can be done using well known formulas [14]. Alternatively one may compute its highest weight vectors and highest weights explicitly using the construction from Section 5.

Let $i: G / P \rightarrow \mathcal{P}\left(\wedge^{2} V^{*}\right)=\mathcal{P}$ be the Plucker embedding and let $\mathcal{O}_{G / P}(1)=i^{*} \mathcal{O P}(1)$ be tautological line bundle on $G / P$ and let $\mathcal{O}_{G}$ ${ }_{P}(d)=\mathcal{O}_{G / P}(1) \otimes^{d}$. It follows from the Borel-Weil-Bott Theorem [16] $\mathrm{H}^{0}(\mathbb{G}, \mathcal{O G}(d))$ is an irreducible $\mathrm{SL}(V)$-module. Let $V$ have basis $e_{1}, e_{2}, e_{3}$, $e_{4}$ and let $\wedge^{2} V$ have basis $e_{i j}$ for $1 \leq i \leq j \leq 4$, with $e_{i j}=e_{i} \wedge e_{j}$. Consider the element $f \in \operatorname{Sym}^{2}\left(\wedge^{2} V\right)$ where

$$
f=e_{12} e_{34}-e_{13} e_{24}+e_{14} e_{23} \text {. }
$$

One checks $f$ is a highest weight vector for $\operatorname{SL}(V)$ with highest weight 0 , hence it defines the unique trivial character of $\operatorname{SL}(V)$. Its dual

$$
f^{*}=x_{12} x_{34}-x_{13} x_{24}+x_{14} x_{23} \in \operatorname{Sym}^{2}\left(\wedge^{2} V^{*}\right)
$$

is the defining equation for $\mathbb{G}=G / P$ as closed subscheme of $\mathcal{P}\left(\wedge^{2} V^{*}\right)$.

Proposition 5.2. The following hold: there is an isomorphism of $\mathrm{SL}(V)$-modules

$$
\begin{aligned}
& \operatorname{Sym}^{d}\left(\wedge^{2} V\right)=\oplus_{i=0}^{l} \mathrm{H}^{0}\left(\mathbb{G}, \mathcal{O}_{\mathbb{G}}(d-2 i)\right)^{*}, \\
& \text { where } l=k \text { if } d=2 k \text { or } d=2 k+1 .
\end{aligned}
$$

Proof. The result is proved using the theory of highest weights. There is a split exact sequence of $\mathrm{SL}(V)$-modules

$$
0 \rightarrow f^{*} \operatorname{Sym}^{d-2}\left(\wedge^{2} V^{*}\right) \rightarrow \operatorname{Sym}^{d}\left(\wedge^{2} V^{*}\right) \rightarrow \mathrm{H}^{0}\left(\mathbb{G}, \mathcal{O}_{\mathbb{G}}(d)\right) \rightarrow 0 .
$$

Dualize this sequence to get the split exact sequence

$$
0 \rightarrow f \operatorname{Sym}^{d-2}\left(\wedge^{2} V\right) \rightarrow \operatorname{Sym}^{d}\left(\wedge^{2} V\right) \rightarrow Q_{d} \rightarrow 0 .
$$

where $Q_{d}=\mathrm{H}^{0}(\mathbb{G}, \mathcal{O} \mathbb{G}(d))^{*}$. Since $f$ is the trivial character it follows there is an isomorphism

$$
f \operatorname{Sym}^{d}\left(\wedge^{2} V\right) \cong \operatorname{Sym}^{d}\left(\wedge^{2} V\right)
$$

of SL $(V)$-modules. By the Borel-Weil-Bott Theorem it follows $Q_{d}$ is an irreducible $\mathrm{SL}(V)$-module. If $d=2 k$ we get by induction the equality

$$
\operatorname{Sym}^{d}\left(\wedge^{2} V^{*}\right)=Q_{d} \oplus Q_{d-2} \oplus \cdots \oplus Q_{2} \oplus Q_{0},
$$

and the claim of the Proposition is proved in the case where $d=2 k$. The claim when $d=2 k+1$ follows by a similar argument and the Proposition is proved.

Corollary 5.3. Let $\mathcal{E}=\oplus_{i=0}^{l} \mathcal{O}_{\mathbb{G}}(2 i-d)$ where $l=k$ if $d=2 k$ or $d=2 k$ +1 . It follows

$\mathrm{H}^{0}(\mathbb{G}, \mathcal{E}) \cong \operatorname{Sym}^{d}\left(\wedge^{2} V^{*}\right)$

as $\mathrm{SL}(V)$-module.

Proof. We get by Proposition 5.4 isomorphisms of SL(V)-modules $\mathrm{H}^{0}(\mathbb{G}, \mathcal{E}) \cong \mathrm{H}^{0}\left(\mathbb{G}, \oplus_{i=0}^{l} \mathcal{O}_{\mathbb{G}}(d-2 i)\right) \cong$

$\oplus_{i=0}^{l} \mathrm{H}^{0}\left(\mathbb{G}, \mathcal{O}_{\mathbb{G}}(d-2 i)\right) \cong \operatorname{Sym}^{d}\left(\wedge^{2} V\right)^{*} \cong \operatorname{Sym}^{d}\left(\wedge^{2} V^{*}\right)$

and the Corollary is proved.

Corollary 5.4. There is for every $d \geq 1$ an equality

$$
\begin{aligned}
& \operatorname{Aut}_{\mathrm{SL}(V)}\left(\operatorname{Sym}^{d}\left(\wedge^{2} V\right)\right)=\prod_{i=0}^{l} K^{*} \\
& \text { where } l=k \text { if } d=2 k \text { or } d=2 k+1 .
\end{aligned}
$$

Proof. This follows from Proposition 5.4 and the Borel-Weil-Bott theorem (BWB). From the BWB theorem it follows $\mathrm{H}^{0}(\mathbb{G}, \mathcal{O O} \mathbb{G}(d))^{*}$ is an irreducible $\operatorname{SL}(V)$-module for all $d \geq 1$. From this and Proposition 5.4 the claim of the Corollary follows.

Hence the $\operatorname{SL}(V)$-module $\operatorname{Sym}^{d}\left(\wedge^{2} V\right)$ is a multiplicity free $\operatorname{SL}(V)$ module for all $d \geq 1$. This is not true in general for $\operatorname{Sym}^{d}\left(\wedge^{m} K^{m+n}\right)$ when $m, n>2$.

In general if $\mathbb{S}_{\lambda}$ and $\mathbb{S}_{\mu}$ are two Schur-Weyl modules [14] there is a decomposition

$$
\mathbb{S}_{\lambda}\left(\mathbb{S}_{\mu}(V)\right) \cong \oplus_{i} V_{\lambda_{i}}
$$

where $V_{\lambda_{i}}$ is an irreducible SL $(V)$-module for all $i$. It is an open problem to calculate this decomposition for two arbitrary partitions $\lambda$ and $\mu$.

\section{Appendix B: The Cauchy Formula}

We include in this section an elementary discussion of the Cauchy formula using multilinear algebra. Let $W \subseteq V$ be vector spaces of dimension $m$ and $m+n$ over $K$ and let $P \subseteq \operatorname{SL}(V)$ be the subgroup fixing $W$. Let $\mathfrak{g}=\operatorname{Lie}(G)$ and $\mathfrak{p}=\operatorname{Lie}(P)$. There is a canonical isomorphism

$$
\mathfrak{g} / \mathfrak{p} \cong \operatorname{Hom}(W, V / W)
$$

of $P$-modules, hence the elements of $\mathfrak{g} / \mathfrak{p}$ may be interpreted as linear maps. The symmetric power $\operatorname{Sym}^{k}(\mathfrak{g} / \mathfrak{p}) \cong \operatorname{Sym}^{k}(\operatorname{Hom}(W, V /$ $W)$ ) is a $P$-module hence a $P_{\text {semi }}=\mathrm{SL}(m) \times \mathrm{SL}(n)$-module and we want to give an explicit construction of its highest weight vectors as $P_{\text {semi }}{ }^{-}$ module.

Proposition 6.1. Let $U=K^{m}$. There is a canonical map of $\operatorname{SL}(V)$ modules 
$\wedge^{m}\left(U^{*}\right) \otimes \wedge^{m} U \rightarrow \operatorname{Sym}^{m}(\operatorname{Hom}(U, U))$

defined by

$x_{1} \wedge \cdots \wedge x_{m} \otimes e_{1} \wedge \cdots \wedge e_{m} \rightarrow\left|\begin{array}{cccc}x_{1} \otimes e_{1} & x_{1} \otimes e_{2} & \cdots & x_{1} \otimes e_{m} \\ x_{2} \otimes e_{1} & x_{2} \otimes e_{2} & \cdots & x_{2} \otimes e_{m} \\ x_{m} \otimes e_{1} & x_{m} \otimes e_{2} & \cdots & x_{m} \otimes e_{m}\end{array}\right|$

Here $e_{1}, . ., e_{m}$ is a basis for $U$ and $x_{1}, . ., x_{m}$ is a basis for $U^{*}$.

Proof. The proof is left to the reader as an exercise.

Note: in Proposition 6.1 the element $x_{i} \otimes e_{j}$ is an element of $U^{*} \otimes U$ $=\operatorname{Hom}(U, U)$. Hence the determinant

$$
\left|\begin{array}{cccc}
x_{1} \otimes e_{1} & x_{1} \otimes e_{2} & \cdots & x_{1} \otimes e_{m} \\
x_{2} \otimes e_{1} & x_{2} \otimes e_{2} & \cdots & x_{2} \otimes e_{m} \\
x_{m} \otimes e_{1} & x_{m} \otimes e_{2} & \cdots & x_{m} \otimes e_{m}
\end{array}\right|
$$

may be interpreted as a polynomial of degree $\mathrm{m}$ in the elements $x_{i} \otimes e_{j}$, hence it is an element of $\operatorname{Sym}^{m}(\operatorname{Hom}(U, U))$.

Let $B \subseteq \mathrm{SL}(m, K) \times \mathrm{SL}(n, K) \subseteq \mathrm{SL}(V)=\mathrm{SL}(m+n, K)$ be the following subgroup: $B$ consists of matrices with determinant one of the form

$$
\left(\begin{array}{cc}
U_{1} & 0 \\
0 & U_{2}
\end{array}\right)
$$

where

$$
U_{1}=\left(\begin{array}{cccc}
a_{11} & 0 & \cdots & 0 \\
a_{21} & a_{22} & \cdots & 0 \\
\vdots & \vdots & \cdots & \vdots \\
a_{m 1} & a_{m 2} & \cdots & a_{m m}
\end{array}\right)
$$

and

$$
U_{2}\left(\begin{array}{cccc}
b_{11} & 0 & \cdots & 0 \\
b_{21} & b_{22} & \cdots & 0 \\
\vdots & \vdots & \cdots & \vdots \\
b_{n 1} & 0 & b_{n 2} \cdots & b_{n n}
\end{array}\right) .
$$

Let $T$ be a $B$-module and $v \in T$ a vector with the property that for all $x \in B$ it follows

$$
x v=\lambda(x) v
$$

where $\lambda \in\left(\operatorname{Hom}\left(B, K^{*}\right)\right.$ is a character of $B$. It follows $v$ is a highest weight vector for $T$ as $\operatorname{SL}(m, K) \times \operatorname{SL}(n, K)$-module. The group $B \subseteq \operatorname{SL}(V)$ defines filtrations of $W$ and $V / W$ as follows: Let $W$ have basis $e_{1}, . ., e_{m}$ and $V$ have basis $e_{1}, . ., e_{m}, f_{1}, . ., f_{n}$. Let $W_{1}=\left\{e_{m}\right\}, W_{2}=\left\{e_{m}, e_{m-1}\right\}$, and

$$
W_{i}=\left\{e_{m}, . . e_{m-i+1}\right\} .
$$

It follows we get a filtration

$$
0=W_{0} \subseteq W_{1} \subseteq \cdots \subseteq W_{m-1}=W
$$

of the vector space $W$. Let

$$
U_{j}=W_{m-1} \cup\left\{f_{n}, . ., f_{n-j+1}\right\}
$$

and let $V_{i}=(V / W) / U_{n-\mathrm{i}}$. We get a surjection

$$
V / W \rightarrow V_{i}
$$

for $i=1, \ldots, n-1$. It follows $\operatorname{dim} W_{i}=\operatorname{dim} V_{i}=d_{i}$ for all $i$. Let $x: W \rightarrow V /$ $W$ be a linear map of vector spaces. We get an induced map

$$
x_{\mathrm{i}}: W_{i} \rightarrow V_{i}
$$

wich is a square $d_{i}$ matrix for all $i$. Let $\mathfrak{g} \in B$ be the element

$\left(\begin{array}{cc}G_{1} & 0 \\ 0 & G_{2}\end{array}\right)$

where

$G_{1}=\left(\begin{array}{cccc}a_{1} & 0 & \cdots & 0 \\ & a_{2} & \cdots & 0 \\ \vdots & \vdots & \cdots & \vdots \\ & * & \cdots & a_{m} \\ & & & \end{array}\right)$

and

$G_{2}\left(\begin{array}{cccc}b_{1} & 0 & \cdots & 0 \\ & b_{2} & \cdots & 0 \\ \vdots & \vdots & \cdots & \vdots \\ & 0 & \cdots & b_{n}\end{array}\right)$.

The $i$ 'th wedge product

$\left|x_{i}\right|=\wedge^{i} x_{i} \in \operatorname{Hom}\left(\wedge^{i} W_{i}, \wedge^{i} V_{i}\right)=\wedge^{i}\left(W_{i}^{*}\right) \otimes \wedge^{i} V_{i}$

may be viewed as an element in

$\left|x_{i}\right| \in \operatorname{Sym}^{i}\left(\operatorname{Hom}\left(W_{i}, V_{i}\right)\right) \subseteq \operatorname{Sym}^{i}(\operatorname{Hom}(W, V / W))$

via Proposition 6.1.

Proposition 6.2. The following formula holds:

$g\left|x_{i}\right|=\frac{b_{1} \cdots b_{i}}{a_{m-i+1} \cdots a_{m}}\left|x_{i}\right|=\lambda(g)\left|x_{i}\right|$

for all $\mathrm{g} \in B$. Here $\lambda(g)=\frac{b_{1} \cdots b_{i}}{a_{m-i+1} \cdots a_{m}}$ is a character $\lambda \in \operatorname{Hom}\left(B, K^{*}\right)$.

Proof. The proof is left to the reader as an exercise.

Hence the $i$ th determinant $\left|x_{i}\right| \in \operatorname{Sym}^{i}(\operatorname{Hom}(W, V / W))$ is a highest weight vector for the $\operatorname{SL}(m) \times \operatorname{SL}(n)$-module $\operatorname{Sym}^{i}(\operatorname{Hom}(W, V / W))$. By the results of studies Brion [17-22], it follows the vectors $x_{0}^{d_{0}} x_{1}^{d_{1}} \cdots x_{i}^{d_{i}}$ with $\sum i d_{i}=k$ are all highest weight vectors for the module

$\operatorname{Sym}^{k}(\operatorname{Hom}(W, V / W)) \cong \operatorname{Sym}^{k}\left(W^{*} \otimes V / W\right)$.

\section{Acknowledgements}

The author thanks Michel Brion, Alexei Roudakov and an anonymous referee for comments on the contents of this paper.

\section{References}

1. Maakestad H (2009) On jet bundles and generalized Verma modules II arXiv:0903: 3291.

2. Maakestad $\mathrm{H}$ (2008) Principal parts on the projective line over arbitrary rings Manuscripta Math 126: 443-464.

3. Maakestad H (2009) Jet bundles on projective space. Travaux Math.

4. Maakestad $\mathrm{H}$ (2004) Modules of principal parts on the projective line. Ark Mat 42: 307-324.

5. Maakestad H (2004) A note on the principal parts on projective space and linear representations. Proc of the AMS 133: 349-355.

6. Mumford D, Fogarty J, Kirwan F (1994) Geometric Invariant Theory. Ergebnisse der Mathematik und ihrer Grenzgebiete, A series of modern surveys in mathematics 34 .

7. Perkinson D (1996) Principal parts of line bundles on toric varieties. Compositio Math 104: 27-39.

8. Piene R, Sacchiero G (1984) Duality for rational normal scrolls. Comm in Alg 12: 1041-1066. 
9. Di Rocco S, Sommese AJ (2001) Line bundles for which a projectivized jet bundle is a product. Proc Amer Math Soc 129: 1659-1663.

10. Sommese $J(1978)$ Compact complex manifolds possessing a line bundle with a trivial jet bundle. Abh Math Sem Univ Hamburg 47: 79-91.

11. Maakestad H (2009) Discriminants of morphisms of sheaves. arXiv: 0911.4804.

12. Lascoux A (1978) Syzygies des varietes determinantales. Advances in math 30.

13. Jantzen JC (2003) Representations of algebraic groups: Math Surveys and Monographs (2nd edn.). American Math Soc 107.

14. Fulton W, Harris J (1991) Representation theory - a first course. Graduate Texts in Mathematics, Springer Verlag.

15. Maakestad $\mathrm{H}$ (2008) On jet bundles and generalized Verma modules. arXiv:0 812: 2751
16. Borel A (1991) Linear algebraic groups. Graduate Texts in Math 126.

17. Brion M (1996) Theory of invariants and geometry quotient varieties.

18. Bögvad R, Källström R (2007) Geometric interplay between function subspaces and their rings of differential operators. Trans Am Math Soc 359: 2075-2108.

19. Dixmier J (1996) Enveloping algebras. Graduate studies in mathematics, American Math Soc 11.

20. Hartshorne R (1977) Algebraic geometry. Graduate Texts in Mathematics 52.

21. Lakshmibai V, Seshadri CS (1991) Standard Monomial Theory. Proceedings of the Hyderabad Conference on Algebraic Groups 1989.

22. Manivel L (1998) Fonctions symetriques, polynomes de Schubert et lieux de degenerescence. Societe Mathematique de France.
This article was originally published in a special issue, Recent Advances of Lie Theory in differential Geometry, in memory of John Nash handled by Editor. Dr. Princy Randriambololondrantomalala, Unversity of Antananarivo, Madagascar

.

